\title{
Viscosity solutions of discontinuous Hamilton-Jacobi equations
}

\author{
XINFU CHEN ${ }^{\dagger}$ \\ Department of Mathematics, University of Pittsburgh, Pittsburgh, PA 15260, USA \\ BEI HU ${ }^{\dagger}$ \\ Department of Mathematics, University of Notre Dame, Notre Dame, IN 46556, USA
}

[Received 31 October 2006 and in revised form 8 November 2007]

\begin{abstract}
We define viscosity solutions for the Hamilton-Jacobi equation $\varphi_{t}=v(x, t) H(\nabla \varphi)$ in $\mathbb{R}^{N} \times(0, \infty)$ where $v$ is positive and bounded measurable and $H$ is non-negative and Lipschitz continuous. Under certain assumptions, we establish the existence and uniqueness of Lipschitz continuous viscosity solutions. The uniqueness result holds in particular for those $v$ which are independent of $t$ and piecewise continuous with discontinuity sets consisting of finitely many smooth lower dimensional surfaces not tangent to each other at any point of their intersection.
\end{abstract}

2000 Mathematics Subject Classification: 35F25, 35A07, 35B30.

Keywords: Hamilton-Jacobi equation; viscosity solution; geometric motion; blow-up; monotonicity.

\section{Introduction}

We consider the initial value problem for the Hamilton-Jacobi equation

$$
\begin{cases}\varphi_{t}(x, t)=v(x, t) H(\nabla \varphi), & x \in \mathbb{R}^{N}, t>0, \\ \varphi(x, 0)=\varphi_{0}(x), & x \in \mathbb{R}^{N}, t=0,\end{cases}
$$

where $v$ is positive, bounded and measurable, and $H(q)$ is non-negative and Lipschitz continuous.

When $H(q)=|q|$, 1.1) can be used to describe the motion of hypersurfaces. In fact, for any constant $c \in \mathbb{R}$, if we denote by $\Gamma(t)$ the level set $\{x: \varphi(\cdot, t)=c\}$ and assume that $\Gamma(t)$ is smooth, then it evolves with a normal velocity equal to $v$. Since in general $\Gamma(t)$ will develop singularities, one needs to introduce weak solutions. The level set approach is to write $\Gamma(t)$ as a level set of a function $\varphi$ and study a $\operatorname{PDE}$ that $\varphi$ satisfies in the whole space-time domain; see, for example [7, 14, 15, 20] and the references therein.

For a weak solution of 1.1 (with $v>0$ ), if one denotes by $\bar{\Omega}^{+}(t)$ and $\Omega^{-}(t)$ the sets where $\varphi(\cdot, t) \geqslant c$ and $\varphi(\cdot, t)<c$, respectively, then $\bar{\Omega}^{+}(t)$ expands with speed $v(x, t)$ in every direction, and $\Omega^{-}(t)$ "yields" to $\bar{\Omega}^{+}(t)$ as little as possible. In general, weak geometric motions are time irreversible, which can be explained as follows.

Consider the shrinking of a square in $\mathbb{R}^{2}$ with velocity $v \equiv 1$. Then, for the geometrical motion corresponding to the viscosity solution of $\varphi_{t}=|\nabla \varphi|$, the square will keep the shape of a square with its sides shrinking with velocity 1 . Note that the corners shrink with speed $\sqrt{2}$, which is what we mean by saying that $\Omega_{t}^{-}$yields as little as possible. In fact, the extra $\sqrt{2}-1$ speed of the corners

\footnotetext{
E-mail: xinfu@pitt.edu
}

E-mail: b1hu@nd.edu 
is gained by the infinite curvature of the square at the corners. This curvature effect is not seen in the differential equation $\varphi_{t}=|\nabla \varphi|$, but is expressed by the definition of viscosity solutions. Now, if we reverse the time and sign of $\varphi$, we will not obtain a corresponding viscosity solution since when a square expands, its corners can only expand with velocity 1 . In fact, the four corners become four quarter circles connecting four line segments identical to the sides of the original square; in this case, $\Omega^{-}(t)$ can yield to the advancement of $\Omega^{+}(t)$ with speed 1 everywhere on the boundary.

When $v$ changes sign one can say that at points where $v>0, \Omega^{-}$resists as much as possible the advancement of $\Omega^{+}$of minimum speed $v$ whereas at points where $v<0, \Omega^{+}$resists as much as it could the expansion of $\Omega^{-}$of minimum speed $|v|$. The definition of viscosity solutions from such an idea may lead to a fattening phenomenon: even if $\Gamma(0)=\{x: \varphi(x, t)=0\}$ is a smooth hypersurface, for some $t>0, \Gamma(t):=\{x: \varphi(x, t)=0\}$ may contain an open set; see [7, 14] for the case of motion by mean curvature. In a physical interpretation, this open set corresponds to a region for which the mathematical model cannot determine which phase the underlying physical system belongs to at time $t$. It is our pessimistic opinion that when $v$ changes sign, one should either abandon the requirement that the solution be unique or supply additional information (more than the "vanishing viscosity") from physics to complete the model.

We shall therefore restrict $v$ to be positive in this paper. The viscosity solution defined in [10, 8] for Hamilton-Jacobi equations then becomes suitable and very powerful here. (For viscosity solutions of second order PDEs, see [9] and the references therein.) However, most works concerning viscosity solutions of first order PDE deal with continuous Hamiltonians. In many applications $([1,2,6,11]$ for example), spatial inhomogeneity often leads to a discontinuous Hamiltonian, and it is not clear at all whether the uniqueness is still valid in this case. We will show, under very general assumptions on $v$, that $(1.1)$ admits a unique viscosity solution.

For any measurable function $v$ defined in a neighborhood of $z \in \mathbb{R}^{m}$, we set

$$
v^{*}(z)=\lim _{\delta \searrow 0} \underset{B_{\delta}(z)}{\operatorname{ess} \sup } v, \quad v_{*}(z)=\lim _{\delta \searrow 0} \underset{B_{\delta}(z)}{\operatorname{essinf}} v
$$

where $B_{\delta}(z)$ represents the ball of radius $\delta$ with center $z$.

Definition 1 Let $\varphi$ be a continuous function on $Q \subset \mathbb{R}^{N} \times[0, \infty)$. We say that $\varphi$ is a supersolution (or subsolution) to $\psi_{t}=v H(\nabla \psi)$ on $Q$, written

$$
\varphi_{t} \geqslant v_{*} H(\nabla \varphi) \quad\left(\text { or } \varphi_{t} \leqslant v^{*} H(\nabla \varphi)\right) \quad \text { in } Q
$$

if for every smooth function $\zeta$ defined on $Q$,

$$
\zeta_{t}\left(z_{0}\right) \geqslant v_{*}\left(z_{0}\right) H\left(\nabla \zeta\left(z_{0}\right)\right) \quad\left(\text { or } \zeta_{t}\left(z_{0}\right) \leqslant v^{*}\left(z_{0}\right) H\left(\nabla \zeta\left(z_{0}\right)\right)\right)
$$

as long as $z_{0}$ is an interior strict local minimum (or maximum) point of $\varphi-\zeta$ in $Q$.

If a function $\varphi$ defined on $\mathbb{R}^{N} \times[0, T)$ is both a supersolution and a subsolution on $\mathbb{R}^{N} \times(0, T)$, then we say that $\varphi$ is a viscosity solution of $\varphi_{t}=v H(\nabla \varphi)$ in $\mathbb{R}^{N} \times(0, T)$ with initial value $\varphi(\cdot, 0)$.

We remark that this definition differs from that of Barron \& Liu [1] who defined semicontinuous viscosity solutions for another class of Hamilton-Jacobi equations.

The idea of using limsup and liminf in defining viscosity solutions for discontinuous Hamiltonians was introduced in Ishii [17], where the equations of the type $u_{t}+H(t, x, u, D u)=0$ were studied. For the uniqueness, Ishii does not require $H$ to be continuous in the variables $t$ 
and $u$, but just integrable in $t$ and monotone in $u$. This differs from our case where the Hamiltonian $v(x) H(p)$ can be discontinuous in the spatial variable.

For the stationary case, the equations such as $\lambda u+H(x, D u)=g(x)$ with the Hamiltonian $H(x, p):=\sup _{a \in A}\{-f(x, a) p-h(x, a)\}$ have received a lot of attention. The functions $f$ and $h$ are assumed to be continuous, but $g(x)$ is not assumed to be continuous. In [21], the discontinuity of $g(x)$ along a smooth curve was allowed; necessary and sufficient conditions for uniqueness were derived with the help of optimality principles for viscosity solutions. In [19], the well-posedness for the equation $H(D u)=g(x)$, where $H$ is convex and $g$ is lower semicontinuous, was established in the class of Monge solutions. Furthermore, its relation to the maximal Lipschitz subsolution is investigated. In [22], the Hamilton-Jacobi equation with $H$ possibly discontinuous in $x$ along a smooth surface, which arises from the shape-from-shading problems in image analysis, was studied; in particular, comparison results (and hence the uniqueness) for subsolutions and supersolutions in the sense of Ishii were established. The concept of Monge solutions was also used in [3] for Hamilton-Jacobi equations of the form $H(x, D u)=0$, where $H(x, p)$ is Borel measurable and quasi-convex in $p$. In [5], the case of less regular Hamiltonians which are just measurable in $x$ was studied.

In [12], the authors studied the problem $H(D u)=g(x)$, where $g$ is allowed to have certain types of discontinuous behavior; the condition used in [12] bears some similarity to the monotonicity property in our Definition 2. The existence and uniqueness of a Lipschitz viscosity solution $u$, as well as a numerical finite difference scheme were presented with an $O(\sqrt{\Delta t})$ error estimate.

For the time-dependent case, the Cauchy problem for an equation of the form $u_{t}+H(x, D u)=0$ was studied in [4], where $H$ is measurable in $x$, continuous, convex and positive homogeneous in $p$. The notion of solution employed in [4] is different from Ishii's; the unique viscosity solution is given by a representation formula of Hopf-Lax type. The assumption differs from ours since we assume neither the positive homogeneity in $p$ nor convexity. Furthermore, their proof is based on establishing the Hopf-Lax type representation formula for viscosity solutions, which is different from our proof.

In the one space dimension case, a problem arising from flame propagation and chemical etching of the form $u_{t}=a(x) \sqrt{1+u_{x}^{2}}$ in $\mathbb{R}^{1} \times(0, \infty)$ was studied in [13]. Here $a(x)$ is only assumed to be bounded and of bounded variation, and to satisfy a one-sided Lipschitz constraint. Existence and uniqueness were established and a finite difference scheme was proved to be convergent with some numerical tests.

Under Definition 1, it is not difficult to establish the existence under appropriate assumptions, even if $v$ is not continuous. The main contribution of this paper is the uniqueness of the viscosity solution in the multi-space dimensional case, under the assumption of either Monotonicity Property or Blow-up Property.

The replacement of $v$ by $v^{*}$ for subsolutions and by $v_{*}$ for supersolutions is very generous. This generosity allows us to establish the existence of viscosity solutions for a large class of $v$.

TheOREM 1 (Existence) Assume that $H$ is continuous and $C^{-1}|q| \leqslant H(q) \leqslant C(1+|q|)$ for all $q \in \mathbb{R}^{N}$ and some $C \geqslant 1$. Also assume that $v$ is uniformly positive and bounded and that for some $m(\cdot) \in L_{\mathrm{loc}}^{\infty}([0, \infty))$ and a.e. $x \in \mathbb{R}^{N}, \ln v(x, t)-m(t)$ is non-increasing in $t \in[0, \infty)$. Then, for every uniformly Lipschitz continuous $\varphi_{0}$, problem 1.1 admits a viscosity solution which is uniformly Lipschitz continuous in $\mathbb{R}^{N} \times[0, T]$ for every $T>0$.

As illustrated by a non-existence example in $\$ 2.5$, even if $v$ is continuous, conditions on the dependence of $v$ on $t$ are still needed for the existence of a continuous viscosity solution. 
Note that a uniformly positive and bounded $v$ satisfies the assumption of the theorem if one of the following holds:

(i) $v$ is a function of $x$ only (take $m \equiv 0$ );

(ii) $v$ is a function of $t$ only (take $m=\ln v$ );

(iii) $(\ln v)_{t} \in L_{\operatorname{loc}}^{1}\left([0, \infty) ; L^{\infty}(\mathbb{R})\right)\left(\operatorname{take} m(t)=\int_{0}^{t}\left\|(\ln v(\cdot, t))_{t}\right\|_{L^{\infty}\left(\mathbb{R}^{N}\right)} \mathrm{d} t\right)$;

(iv) There exists a decomposition $[0, \infty)=\bigcup_{j=1}^{\infty}\left[t_{j-1}, t_{j}\right.$ ) such that $v$ satisfies (i) or (ii) or (iii) in each interval $\left(t_{j-1}, t_{j}\right), j=1,2, \ldots$.

Our generous replacement of $v$ in the definition of viscosity solutions, on the other hand, invalidates the uniqueness of the viscosity solutions in some cases. For example, when the set where $v^{*} \neq v_{*}$ has a positive measure, it is easy to construct non-uniqueness examples; see $\S 2$.6. Since quite often one of the inequalities in $(1.2)$ is actually an equality at points where $v_{*}<v^{*}$, it is not clear how to modify our definition to get uniqueness when the set $v^{*} \neq v_{*}$ has a positive measure.

Nevertheless, we are still able to prove uniqueness for a large class of $v$. In particular, our uniqueness result holds if $v$ has the following monotonicity property at every point in $\mathbb{R}^{N} \times(0, \infty)$.

Definition 2 A function $v$ is said to have the monotonicity property at $\left(x_{0}, t_{0}\right) \in \mathbb{R}^{N} \times(0, \infty)$ if there exists a unit vector $\vec{e} \in \mathbb{R}^{N+1}$ such that, for $K=\operatorname{ess} \sup v$,

$$
\lim _{\rho \searrow 0} \sup _{\left|y-x_{0}\right| \leqslant K\left(t_{0}-\tau\right) \leqslant M \rho}\left[v^{*}(y, \tau)-v_{*}((y, \tau)-\rho \vec{e})\right] \leqslant 0 \quad \forall M>1 .
$$

Clearly, the monotonicity property holds at points where $v$ is continuous. It also holds if $v$ has only jump discontinuities and its discontinuity set is a continuous graph; namely, at any point $z_{0}$ at which $v$ is not continuous, there exist $\delta>0$ and a continuous function $f$ such that, after a rotation, $v$ is uniformly continuous in $\left\{z:\left|z-z_{0}\right| \leqslant \delta, \pm\left(z_{N+1}-f\left(z_{1}, \ldots, z_{N}\right)\right)>0\right\}$.

Our uniqueness theorem also includes another class of functions having the following blow-up property:

DEFINITION 3 A function $v$ defined in a neighborhood of $z_{0} \in \mathbb{R}^{m}$ is said to have a first order blow-up at $z_{0}$ if $v^{(1)}\left[z_{0}\right](\cdot):=\lim _{\lambda \downarrow 0} v\left(z_{0}+\lambda \cdot\right)$ exists a.e. in $\mathbb{R}^{m}$ and for every $e \in \mathbb{R}^{m}$,

$$
\left(v^{(1)}\left[z_{0}\right]\right)_{*}(e)=\liminf _{\lambda \searrow 0, z \rightarrow e} v_{*}\left(z_{0}+\lambda z\right), \quad\left(v^{(1)}\left[z_{0}\right]\right)^{*}(e)=\limsup _{\lambda \searrow 0, z \rightarrow e} v^{*}\left(z_{0}+\lambda z\right) .
$$

The $(k+1)$-th order blow-up $v^{(k+1)}\left[z_{0}, e_{1}, \ldots, e_{k}\right](\cdot)$ is the first order blow-up, if it exists, of the $k$-th order blow-up $v^{(k)}\left[z_{0}, e_{1}, \ldots, e_{k-1}\right](\cdot)$ at $e_{k}$.

A function $v$ is said to have the blow-up property at $z_{0}$ if $v^{(k)}\left[z_{0}, e_{1}, \ldots, e_{k-1}\right](\cdot)$ exists for all $k \geqslant 1$ and all $e_{1}, \ldots, e_{k-1} \in \mathbb{R}^{m}$.

We remark that the monotonicity property and the blow-up property are two totally different concepts; in general one does not imply the other. For example, on $\mathbb{R}^{2}$ define $v(x, y)=2$ for $y>x \sin (1 / x)$ and $v=1$ for $y \leqslant x \sin (1 / x)$; then at the origin, $v$ has the monotonicity property but does not even have a first order blow-up. On the other hand, define $v=1$ when $x y>0$ and $v=2$ when $x y \leqslant 0$; then $v$ has the blow-up property but not the monotonicity property.

We shall study in great detail the blow-up property in $\S 5$. Here we only point out the following. Blow-ups are homogeneous of degree zero; namely, writing $v^{(k)}\left[z_{0}, e_{1}, \ldots, e_{k-1}\right](\cdot)$ as $v^{(k)}(\cdot)$, we have $v^{(k)}(\lambda e)=v^{(k)}(e)$ for every $\lambda>0$ and a.e. $e \in \mathbb{R}^{m}$. With this homogeneity, a blow-up "reduces" the space dimension that the original function depends on. In fact, as shown in $\S 5$, if $e_{1}, \ldots, e_{k}(1 \leqslant k \leqslant m)$ are the coordinate directions of $x_{1}, \ldots, x_{k}$, respectively 
$\left(z=\left(x_{1}, \ldots, x_{m}\right)\right)$, then the $(k+1)$-th order blow-up $v^{(k+1)}\left[z_{0}, e_{1}, \ldots, e_{k}\right](\cdot)$ (if it exists) depends only on $\left(x_{k+1}, \ldots, x_{m}\right)$. In particular, $v^{(m+1)}\left[z_{0}, e_{1}, \ldots, e_{m}\right](\cdot)$ is a constant function.

It is easy to show that $v(z)$ has the blow-up property if (i) $v(z)$ is piecewise uniformly continuous, and (ii) the set $J$ where $v$ is discontinuous consists of only finitely many smooth lower dimensional surfaces which are not tangent to each other at any point of their intersection. As an illustration, we provide two examples that satisfy (i) and (ii).

EXAmPLE 1.1 In $\mathbb{R}^{2}$, set $v(z)=c_{i}$ in $\Omega_{i}, i=1, \ldots, K$, where $K \geqslant 2$, all $c_{1}, \ldots, c_{K}$ are constants, and $\Omega_{1}, \ldots, \Omega_{K}$ are domains divided by $C^{1}$ closed curves $\ell_{1}, \ldots \ell_{K}$ which do not intersect each other except at the origin where they all meet non-tangentially.

EXAMPLE 1.2 In $\mathbb{R}^{3}$, let $v$ be any positive function which is uniformly continuous in each of the eight octants, but discontinuous across any coordinate planes. Note that the discontinuity set $J$ is the union of all the coordinate planes, which can be divided into $J_{2} \cup J_{1} \cup J_{0}$ where $J_{2}$ is the union of twelve open 2-D surfaces (quadrants of the coordinate planes), $J_{1}$ is the union of six open 1-D rays (halves of the coordinate lines), and $J_{0}$ is the origin.

If $z_{0} \notin J$, then $v^{(1)}\left[z_{0}\right](\cdot)$ is a constant function.

If $z_{0} \in J_{2}$, say $z_{0}=(0,1,1)$, then $v^{(1)}\left[z_{0}\right]((x, y, z))$ is constant for $x>0$ and for $x<0$.

If $z_{0} \in J_{1}$, say, $z_{0}=(0,0,1)$, then $v^{(1)}\left[z_{0}\right]((x, y, z))$ is a function of $(x, y)$ and is constant in each of the four quadrants in the $x-y$ plane. A further blow-up in the direction $(0,1,0)$ then gives a function which is constant for $x>0$ and for $x<0$.

If $z_{0}=(0,0,0)$, then $v^{(1)}\left[z_{0}\right](\cdot)$ is a function equal to a constant in each of the eight octants in $\mathbb{R}^{3}$. Further blow-ups of $v^{(1)}[(0,0,0)](\cdot)$ follow the same pattern as what we just did for $v$ itself.

When the discontinuity set of $v$ has a cusp, it may not have the blow-up property.

EXAmple 1.3 In $\mathbb{R}^{2}$, let $v(x, y)=2$ when $|y| \leqslant x^{2}$ and $v=1$ otherwise. Then the first order blow-up of $v$ at the origin does not exist. In fact, $w(e)=\lim _{\lambda \searrow 0} v(\lambda e)$ exists for all $e \neq(0,0)$, but it does not satisfy $[1.4$ for $e=(1,0)$. Since $w \equiv 1$ a.e., $w$ does not carry all the information concerning sup and inf of $v$ in all cones with vertex $(0,0)$.

Our uniqueness theorem is as follows.

THEOREM 2 (Uniqueness) Let $H(q)$ be non-negative, uniformly Lipschitz continuous, and nondecreasing in $|q|$, and let $v$ be non-negative and uniformly bounded. Assume that for every $z_{0} \in$ $\mathbb{R}^{N} \times(0, \infty)$, either (i) $v$ has the monotonicity property at $z_{0}$, or (ii) $v$ has the blow-up property at $z_{0}$ and $v^{(1)}\left[z_{0}\right](x, t)$ is independent of $t$. Then Lipschitz continuous viscosity solutions to 1.1 are unique.

In $\S 2$, we prove various properties of the viscosity solutions and give a few examples which illustrate the difficulties in studying the existence, uniqueness and continuous dependence of solutions of (1.1). In $\$ 3$, we prove the existence Theorem 1 In $\S 4$ we prove uniqueness under the monotonicity assumption on $v$. In $\S 5$, we discuss the blow-up property and provide a few more examples. Finally, in $\S 6$, we prove a uniqueness theorem slightly stronger than Theorem 2 .

\section{Some properties and examples of viscosity solutions}

\subsection{Continuous dependence}

It is well-known that for a Hamiltonian equation $u_{t}=G(x, t, \nabla u)$ with a continuous $G$, the continuous limit of a family of uniformly continuous viscosity solutions is still a viscosity solution. 
This is still true with our definition of viscosity solutions (cf. Corollary 4 below). Also, if $G_{n}$ is a sequence of continuous functions converging uniformly to $G$ in any compact set of $(x, t, p) \in$ $\mathbb{R}^{N} \times[0, \infty) \times \mathbb{R}^{N}$, then the limit of viscosity solutions with respect to $\left\{G_{n}\right\}$ is also a viscosity solution of the limit Hamiltonian equation with respect to $G$. As shown by an example later in $\S 2.4$, when $G_{n}$ converges to a discontinuous $G$ (so the convergence is not uniform), the conclusion may not be true.

LEMma 3 Let $H(\cdot)$ be non-negative and continuous, $Q$ be an open set, and $\left\{\psi_{j}\right\}_{j=1}^{\infty}$ be a sequence of viscosity supersolutions to $\left(\psi^{j}\right)_{t} \geqslant\left(v^{j}\right)_{*} H\left(\nabla \psi^{j}\right)$ in $Q$. Assume that, for some $\psi$ and $v$, $\lim _{j \rightarrow \infty} \psi^{j}=\psi$ uniformly in $Q$, and

$$
v_{*}(x, t) \leqslant \liminf _{j \rightarrow \infty,(y, \tau) \rightarrow(x, t)}\left(v^{j}\right)_{*}(y, \tau) \quad \text { for every }(x, t) \in Q .
$$

Then $\psi_{t} \geqslant v_{*} H(\nabla \psi)$ in $Q$. An analogous conclusion also holds for subsolutions.

Proof. Assume that $\psi-\zeta$ attains a strict local minimum at $\left(x_{0}, t_{0}\right) \in Q$ for some smooth $\zeta$. Then, for all sufficiently large $j$, the function $\psi^{j}-\zeta$ attains a local minimum at $\left(x_{j}, t_{j}\right) \in Q$ which satisfies $\lim _{j \rightarrow \infty}\left(x_{j}, t_{j}\right)=\left(x_{0}, t_{0}\right)$. Since $\psi^{j}$ is a supersolution, $\zeta_{t}\left(x_{j}, t_{j}\right) \geqslant\left(v^{j}\right)_{*}\left(x_{j}, t_{j}\right) H\left(\nabla \zeta\left(x_{j}, t_{j}\right)\right)$. Letting $j \rightarrow \infty$ we then obtain $\zeta_{t}\left(x_{0}, t_{0}\right) \geqslant v_{*}\left(x_{0}, t_{0}\right) H\left(\nabla \zeta\left(x_{0}, t_{0}\right)\right)$.

COROLlary 4 Let $H(\cdot)$ be non-negative and continuous, $Q$ be an open set, and $\left\{\psi_{j}\right\}_{j=1}^{\infty}$ be a sequence of viscosity solutions to $\left(\psi^{j}\right)_{t}=v^{j} H\left(\nabla \psi^{j}\right)$ in $Q$. Assume that there exist $v$ and $\psi$ such that $\lim _{j \rightarrow \infty} \psi^{j}=\psi$ uniformly $Q$ and

$$
\begin{array}{ll}
v^{*}(x, t) \geqslant \limsup _{j \rightarrow \infty,(y, \tau) \rightarrow(x, t)}\left(v^{j}\right)^{*}(y, \tau) & \text { for every }(x, t) \in Q, \\
v_{*}(x, t) \leqslant \liminf _{j \rightarrow \infty,(y, \tau) \rightarrow(x, t)}\left(v^{j}\right)_{*}(y, \tau) & \text { for every }(x, t) \in Q .
\end{array}
$$

Then $\psi_{t}=v H(\nabla \psi)$ in $Q$.

Note that 2.1 and 2.2 are obviously valid if $v^{j}=v$ for all $j$.

Here we require 2.1) and 2.2) to be true for every $(x, t)$ in $Q$, not just for almost all points $(x, t) \in Q$. This condition cannot be weakened, as demonstrated by an example in $\S 2.4$.

\subsection{Restriction of supersolutions and subsolutions to lower space dimensions}

LEMma 5 Let $H(\cdot)$ be continuous and non-negative, $k \in\{1, \ldots, N\}$ be any integer, $D$ be an open set in $\mathbb{R}^{k}$, and $Q$ be an open set in $\mathbb{R}^{N-k} \times(0, \infty)$. Then the following holds:

(1) Assume that $\varphi$ is a viscosity subsolution to $\varphi_{t} \leqslant v^{*}(x, t) H(\nabla \varphi)$ in $D \times Q$ and that for any $\left(x^{\prime \prime}, t\right) \in Q$, the function $\varphi\left(\cdot, x^{\prime \prime}, t\right)$ is Lipschitz continuous with Lipschitz constant $\eta$; namely, $\sqrt{\varphi_{x_{1}}^{2}+\cdots+\varphi_{x_{k}}^{2}} \leqslant \eta$ a.e. in $D \times Q$. Then for every $a \in D$, the function $\psi\left(x^{\prime \prime}, t\right):=\varphi\left(a, x^{\prime \prime}, t\right)$ is a viscosity subsolution to

$$
\psi_{t}\left(x^{\prime \prime}, t\right) \leqslant v^{*}\left(a, x^{\prime \prime}, t\right) \bar{H}\left(\nabla_{x^{\prime \prime}} \psi\right), \quad\left(x^{\prime \prime}, t\right) \in Q,
$$

where

$$
\bar{H}\left(p^{\prime \prime}\right):=\max \left\{H\left(p^{\prime}, p^{\prime \prime}\right):\left|p^{\prime}\right| \leqslant \eta\right\} \quad \forall p^{\prime \prime} \in \mathbb{R}^{N-k}
$$


(2) Assume that $\varphi(x, t)$ is a viscosity supersolution to $\varphi_{t} \geqslant v_{*}(x, t) H(\nabla \varphi)$ in $D \times Q$ and that

$$
\lim _{|p| \rightarrow \infty} H(p)=+\infty
$$

Then for every $a \in D$, the function $\psi\left(x^{\prime \prime}, t\right):=\varphi\left(a, x^{\prime \prime}, t\right)$ is a viscosity supersolution to

$$
\psi_{t}\left(x^{\prime \prime}, t\right) \geqslant v_{*}\left(a, x^{\prime \prime}, t\right) \underline{H}\left(\nabla_{x^{\prime \prime}} \psi\right), \quad\left(x^{\prime \prime}, t\right) \in Q,
$$

where

$$
\underline{H}\left(p^{\prime \prime}\right):=\min \left\{H\left(p^{\prime}, p^{\prime \prime}\right): p^{\prime} \in \mathbb{R}^{k}\right\} \quad \forall p^{\prime \prime} \in \mathbb{R}^{N-k} .
$$

Due to the non-trivial difference in the definition of $\bar{H}$ and $\underline{H}$, we do not require the Lipschitz continuity of $\varphi$ in the second assertion of the lemma.

Proof. (1) Let $\zeta\left(x^{\prime \prime}, t\right)$ be a smooth function and assume that $\psi-\zeta$ takes a strict local maximum at $\left(x_{0}^{\prime \prime}, t_{0}\right)$. Then for sufficiently small positive $\varepsilon$, the function $W\left(x^{\prime}, x^{\prime \prime}, t\right)=\varphi\left(x^{\prime}, x^{\prime \prime}, t\right)-\zeta\left(x^{\prime \prime}, t\right)$ $-(1 / \varepsilon)\left|x^{\prime}-a\right|^{2}$ takes a local maximum at some $\left(x_{\varepsilon}^{\prime}, x_{\varepsilon}^{\prime \prime}, t_{\varepsilon}\right)$ which satisfies $\lim _{\varepsilon \rightarrow 0+}\left(x_{\varepsilon}^{\prime}, x_{\varepsilon}^{\prime \prime}, t_{\varepsilon}\right)=$ $\left(a, x_{0}^{\prime \prime}, t_{0}\right)$. Using $W\left(a+s\left(x_{\varepsilon}^{\prime}-a\right), x_{\varepsilon}^{\prime \prime}, t_{\varepsilon}\right) \leqslant W\left(x_{\varepsilon}^{\prime}, x_{\varepsilon}^{\prime \prime}, t_{\varepsilon}\right)$ for $s \in(0,1)$, we have

$$
\frac{1}{\varepsilon}\left(1-s^{2}\right)\left|x_{\varepsilon}^{\prime}-a\right|^{2} \leqslant \varphi\left(x_{\varepsilon}^{\prime}, x_{\varepsilon}^{\prime \prime}, t_{\varepsilon}\right)-\varphi\left(a+s\left(x_{\varepsilon}^{\prime}-a\right), x_{\varepsilon}^{\prime \prime}, t_{\varepsilon}\right) \leqslant(1-s) \eta\left|x_{\varepsilon}^{\prime}-a\right|
$$

since $\varphi\left(\cdot, x_{\varepsilon}^{\prime \prime}, t_{\varepsilon}\right)$ is Lipschitz continuous with Lipschitz constant $\eta$. Letting $s \nearrow 1$, we find that $\left|x_{\varepsilon}^{\prime}-a\right| \leqslant \varepsilon \eta / 2$.

As $\varphi$ is a subsolution,

$$
\begin{aligned}
\zeta_{t}\left(x_{\varepsilon}^{\prime \prime}, t_{\varepsilon}\right) & \leqslant v^{*}\left(x_{\varepsilon}^{\prime}, x_{\varepsilon}^{\prime \prime}, t_{\varepsilon}\right) H\left(\frac{2\left(x_{\varepsilon}^{\prime}-a\right)}{\varepsilon}, \nabla_{x^{\prime \prime}} \zeta\left(x_{\varepsilon}^{\prime \prime}, t_{\varepsilon}\right)\right) \\
& \leqslant v^{*}\left(x_{\varepsilon}^{\prime}, v_{\varepsilon}^{\prime \prime}, t_{\varepsilon}\right) \bar{H}\left(\nabla_{x^{\prime \prime}} \zeta\left(x_{\varepsilon}^{\prime \prime}, t_{\varepsilon}\right)\right)
\end{aligned}
$$

Letting $\varepsilon \searrow 0$ and using the upper semicontinuity of $v^{*}$ we immediately obtain $\zeta_{t}\left(x_{0}^{\prime \prime}, t_{0}\right) \leqslant$ $v^{*}\left(a, x_{0}^{\prime \prime}, t_{0}\right) \bar{H}\left(\nabla_{x^{\prime \prime}} \zeta\left(x_{0}^{\prime \prime}, t_{0}\right)\right)$. This proves (1).

The proof of (2) is similar except that the estimate of $\left|x_{\varepsilon}^{\prime}-a\right|$ is not needed.

REMARK 2.1 The condition 2.3 ensures the minimum in 2.4 is attained. When working on Lipschitz supersolutions, we can always modify $H(p)$ for $|p| \gg 1$ so that 2.3 is satisfied.

\subsection{Transformation}

LEMMA 6 Let $Q$ be an open set in $\mathbb{R}^{N} \times(0, \infty)$ and $\varphi$ be a subsolution to $\varphi_{t} \leqslant v^{*} H(\nabla \varphi)$ in $Q$.

(1) If $\widetilde{H}(p) \geqslant H(p)$ for all $p \in \mathbb{R}^{N}$, then $\varphi_{t} \leqslant v^{*} \tilde{H}(\nabla \varphi)$ in $Q$.

(2) If $F: \mathbb{R}^{N} \rightarrow \mathbb{R}^{N}$ is a diffeomorphism and $b$ is a constant, then $\psi(x, t):=\varphi(F(x), t)+b t$ is a subsolution, in its definition domain, to

$$
\psi_{t}(x, t) \leqslant v^{*}(F(x), t) H\left(\nabla \psi(x, t)\left(D_{x} F\right)^{-1}(x)\right)+b .
$$

A similar assertion also holds for viscosity supersolutions.

The assertion follows directly from the definition of viscosity subsolutions and supersolutions. 


\subsection{An unstable example}

Consider, in one space dimension, the viscosity solution of the initial value problem

$$
\left\{\begin{array}{l}
\varphi_{t}=v(x) \sqrt{1+\left|\varphi_{x}\right|^{2}}, \quad x \in \mathbb{R}, t>0 \\
\varphi(x, 0) \equiv 0, \quad x \in \mathbb{R}
\end{array}\right.
$$

If $v(x) \equiv 1$, then $\varphi(x, t) \equiv t$ is the unique viscosity solution of the problem.

Consider now the case

$$
v_{\varepsilon}(x)= \begin{cases}2 & \text { if }|x| \leqslant \varepsilon \\ 1 & \text { if }|x|>\varepsilon\end{cases}
$$

The solution is unique (by Theorem 7 in $\S 4$ ), and is given by

$$
\varphi_{\varepsilon}(x, t)= \begin{cases}2 t & \text { when }|x| \leqslant \varepsilon \\ 2 t-\sqrt{3}(|x|-\varepsilon) & \text { when } \varepsilon<|x| \leqslant \varepsilon+t / \sqrt{3} \\ t & \text { when }|x|>\varepsilon+t / \sqrt{3}\end{cases}
$$

It is clear that

$$
\begin{aligned}
& \lim _{\varepsilon \rightarrow 0+} \varphi_{\varepsilon}(x, t)=\varphi_{0}(x, t):=\left\{\begin{array}{ll}
2 t-\sqrt{3}|x|, & |x| \leqslant t / \sqrt{3}, \\
t, & |x|>t / \sqrt{3},
\end{array} \quad\right. \text { uniformly, } \\
& \lim _{\varepsilon \rightarrow 0+} v_{\varepsilon}(x)=v_{0}(x) \equiv 1 \quad \text { except at } x=0 .
\end{aligned}
$$

However, by our definition, $\varphi_{0}(x, t)$ is not a viscosity solution with respect to $v_{0}$.

The reason for this is that the set where $v_{\varepsilon}(x)$ equals 2 shrinks to a point, and the condition (2.1) does not hold at the point $x=0$.

\subsection{A non-existence example}

If $v$ is independent of $t$ and is uniformly positive and bounded, our existence Theorem 1 guarantees the existence of a Lipschitz continuous solution. Nevertheless, if $v$ depends on $t$, even if $v$ is continuous, continuous solutions may not exist, as shown by the following example.

In $\mathbb{R}^{1}$, let $H(p)=|p|$ and $v(x, t)=c(x+t)$ where $c(\cdot)$ is any continuous and non-decreasing function satisfying $c(\cdot)=1$ in $(-\infty, 0]$ and $\int_{0}^{1} \mathrm{~d} s /(c(s)-1)<\infty$. Assume that the initial data $\varphi_{0}(x)=\varphi(x, 0)$ is smooth and strictly increasing. Then one can show that $\varphi_{x}(x, t) \geqslant 0$ in the distribution sense, so that $\varphi_{t}=v\left|\varphi_{x}\right|=c(x+t) \varphi_{x}$. This is a linear equation having a unique solution which can be obtained by the method of characteristic curves. If we denote by $x=X\left(x_{0}, t\right)$ the characteristic curve starting from $x_{0}$, then $X_{t}=-c(X+t)$, so that $X(0, t)=-t$ and $\int_{x_{0}}^{X+t} \mathrm{~d} s /(c(s)-1)=-t$ for $x_{0}>0$. Since $\int_{0}^{1} \mathrm{~d} s /(c(s)-1)<\infty$, the characteristic curves $x=X(0, t)$ and $x=X\left(x_{0}, t\right)\left(x_{0}>0\right)$ become the same after time $\int_{0}^{x_{0}} \mathrm{~d} s /(c(s)-1)$. Therefore, a shock wave occurs at the line $x=-t$, and the solution $\varphi$ cannot be continuous.

\subsection{A solution formula for piecewise constant $v$}

In this subsection, we construct a viscosity solution to the initial value problem

$$
\varphi_{t}=v(x)|\nabla \varphi| \quad \text { in } \mathbb{R}^{N} \times(0, \infty), \quad \varphi(\cdot, 0)=g(\cdot) \quad \text { on } \mathbb{R}^{N}
$$


where $g$ is a continuous function, and $v(x)$ is given by

$$
v(x)= \begin{cases}1 & \text { if } x \in Q_{1}, \\ 2 & \text { if } x \in Q_{2}, \\ v_{J} & \text { if } x \in J:=\mathbb{R}^{N} \backslash\left(Q_{1} \cup Q_{2}\right),\end{cases}
$$

where $Q_{1}$ and $Q_{2}$ are open disjoint sets such that $\mathbb{R}^{N}=\bar{Q}_{1} \cup \bar{Q}_{2}$, and $v_{J} \in(1,2)$ is a constant.

Geometrically, for every $c \in \mathbb{R}$, if we denote by $\bar{\Omega}_{t}^{c}$ the set where $\varphi(\cdot, t) \geqslant c$, then $\bar{\Omega}_{t}^{c}$ expands in every direction with speed $v$. In other words, the set $\bar{\Omega}_{t}^{c}$ contains those (and only those) points which can be reached by traveling from some point in $\bar{\Omega}_{0}^{c}$ with speed $v(x)$ at every point $x$ one passes, and in time no longer than $t$.

Based on this geometrical interpretation, we now construct a viscosity solution to 2.5. We first define the modified length of a smooth curve $\gamma=\{X(s): s \in[0,1]\}$ by setting $\ell(\gamma)=$ $\int_{0}^{1}\left(\left|X^{\prime}(s)\right| / v(X(s))\right) \mathrm{d} s$. Note that the function $v(X(\cdot))$ is measurable since it equals 1 in an open set, 2 in another open set, and $v_{J}$ in the remaining set. We define the modified distance $\widehat{d}(x, y)$ from $x$ to $y$ by

$$
\widehat{d}(x, y)=\inf \{\ell(\gamma): \gamma \text { connects } x \text { and } y \text { and is smooth }\} .
$$

Note that $\widehat{d}(x, y)$ is the minimum time needed to travel from $x$ to $y$ with speed $v$.

Since both $Q_{1}$ and $Q_{2}$ are open and $v_{J} \in(1,2)$, one can show that for any $x_{0}$, there exists an open ball $B_{r}\left(x_{0}\right)$ such that $v_{*}\left(x_{0}\right) \leqslant v(x) \leqslant v^{*}\left(x_{0}\right)$ for all $x \in B_{r}\left(x_{0}\right)$. Consequently,

$$
v_{*}\left(x_{0}\right) \widehat{d}\left(x_{0}, x\right) \leqslant\left|x_{0}-x\right| \leqslant v^{*}\left(x_{0}\right) \widehat{d}\left(x_{0}, x\right) \quad \forall x \in B_{r\left(x_{0}\right)}\left(x_{0}\right) .
$$

We claim that the function defined below is a viscosity solution to 2.5 :

$$
\varphi(x, t)=\max \{g(y): \widehat{d}(x, y) \leqslant t\} \quad \forall x \in \mathbb{R}^{N}, t \geqslant 0 .
$$

Proof. First we show that $\varphi$ is a supersolution. Suppose that for some smooth $\zeta, \varphi-\zeta$ attains a strict local minimum at $\left(x_{0}, t_{0}\right) \in \mathbb{R}^{N} \times(0, \infty)$.

If the maximum of $g$ in $\left\{y: \widehat{d}\left(x_{0}, y\right) \leqslant t_{0}\right\}$ cannot be obtained at the boundary, then $\varphi$ is a constant in a neighborhood of $\left(x_{0}, t_{0}\right)$ so there is nothing to show. Thus, we assume that $\varphi\left(x_{0}, t_{0}\right)=$ $g\left(y_{0}\right)$ for some $y_{0}$ satisfying $\widehat{d}\left(x_{0}, y_{0}\right)=t_{0}$. Let $\vec{n}$ be a unit vector such that $\left|\nabla \zeta\left(x_{0}, t_{0}\right)\right|=\vec{n}$. $\nabla \zeta\left(x_{0}, t_{0}\right)$.

For every small $\varepsilon>0$, let $x_{\varepsilon}=x_{0}+\varepsilon v_{*}\left(x_{0}\right) \vec{n}$. We claim that $\varphi\left(x_{\varepsilon}, t_{0}-\varepsilon\right) \leqslant \varphi\left(x_{0}, t_{0}\right)$. It suffices to show that $\left\{y: \widehat{d}\left(x_{\varepsilon}, y\right) \leqslant t_{0}-\varepsilon\right\} \subset\left\{y: \widehat{d}\left(x_{0}, y\right) \leqslant t_{0}\right\}$. In fact, if $\widehat{d}\left(x_{\varepsilon}, y\right) \leqslant t_{0}-\varepsilon$, then $\widehat{d}\left(x_{0}, y\right) \leqslant \widehat{d}\left(x_{\varepsilon}, y\right)+\widehat{d}\left(x_{0}, x_{\varepsilon}\right) \leqslant t_{0}-\varepsilon+\widehat{d}\left(x_{0}, x_{\varepsilon}\right)$. In view of $2.77, \widehat{d}\left(x_{0}, x_{\varepsilon}\right) \leqslant\left|x_{0}-x_{\varepsilon}\right| / v_{*}\left(x_{0}\right)$ $\leqslant \varepsilon$, so that $\widehat{d}\left(x_{0}, y\right) \leqslant t_{0}$. This proves the claim.

We can now calculate

$$
\begin{aligned}
& \zeta_{t}\left(x_{0}, t_{0}\right)-v_{*}\left(x_{0}\right)\left|\nabla \zeta\left(x_{0}, t_{0}\right)\right|=\zeta_{t}\left(x_{0}, t_{0}\right)-v_{*}\left(x_{0}\right) \vec{n} \cdot \nabla \zeta\left(x_{0}, t_{0}\right) \\
& \quad=\frac{1}{\varepsilon}\left\{\zeta\left(x_{0}, t_{0}\right)-\zeta\left(x_{\varepsilon}, t_{0}-\varepsilon\right)\right\}+O(\varepsilon) \geqslant \frac{1}{\varepsilon}\left\{\varphi\left(x_{0}, t_{0}\right)-\varphi\left(x_{\varepsilon}, t_{0}-\varepsilon\right)\right\}+O(\varepsilon) \geqslant O(\varepsilon) .
\end{aligned}
$$

Sending $\varepsilon$ to zero we then conclude that $\varphi$ is a viscosity supersolution.

Now we show that $\varphi$ is a subsolution. Let $\zeta$ be a smooth function such that $\varphi-\zeta$ attains a strict local maximum at $\left(x_{0}, t_{0}\right) \in \mathbb{R}^{N} \times(0, \infty)$. As before, we need only consider the case where the maximum of $g$ on $\left\{y: d\left(x_{0}, y\right) \leqslant t_{0}\right\}$ is attained at some point $y_{0}$ with $d\left(x_{0}, y_{0}\right)=t_{0}$. 
For every sufficiently small $\varepsilon$, let $x_{\varepsilon}$ be a point such that $\widehat{d}\left(x_{0}, x_{\varepsilon}\right)=\varepsilon$ and $\widehat{d}\left(x_{0}, y_{0}\right)=$ $\widehat{d}\left(x_{0}, x_{\varepsilon}\right)+\widehat{d}\left(x_{\varepsilon}, y_{0}\right)$. Then $\widehat{d}\left(x_{\varepsilon}, y_{0}\right)=t_{0}-\varepsilon$ so that, by definition, $\varphi\left(x_{\varepsilon}, t_{0}-\varepsilon\right) \geqslant g\left(y_{0}\right)=$ $\varphi\left(x_{0}, t_{0}\right)$.

Let

$$
\vec{n}_{\varepsilon}=\frac{x_{\varepsilon}-x_{0}}{v^{*}\left(x_{0}\right) \varepsilon}=\frac{x_{\varepsilon}-x_{0}}{v^{*}\left(x_{0}\right) \widehat{d}\left(x_{\varepsilon}, x_{0}\right)} .
$$

Then $x_{\varepsilon}=x_{0}+\varepsilon v^{*}\left(x_{0}\right) \vec{n}_{\varepsilon}$, and in view of 2.7$),\left|\vec{n}_{\varepsilon}\right| \leqslant 1$. Consequently,

$$
\begin{aligned}
\zeta_{t}\left(x_{0}, t_{0}\right) & -v^{*}\left(x_{0}\right)\left|\nabla \zeta\left(x_{0}, t_{0}\right)\right| \leqslant \zeta\left(x_{0}, t_{0}\right)-v^{*}\left(x_{0}\right) \vec{n}_{\varepsilon} \cdot \nabla \zeta\left(x_{0}, t_{0}\right) \\
& =\frac{\zeta\left(x_{0}, t_{0}\right)-\zeta\left(x_{\varepsilon}, t_{0}-\varepsilon\right)}{\varepsilon}+O(\varepsilon) \leqslant \frac{\varphi\left(x_{0}, t_{0}\right)-\varphi\left(x_{\varepsilon}, t_{0}-\varepsilon\right)}{\varepsilon}+O(\varepsilon) \leqslant O(\varepsilon) .
\end{aligned}
$$

Sending $\varepsilon \searrow 0$ we then obtain the required inequality for $\varphi$ being a subsolution.

REMARK 2.2 Note that, by 2.8 ,

$$
\bar{\Omega}_{t}^{c}:=\{x: \varphi(x, t) \geqslant c\}=\{x: \exists y, \widehat{d}(x, y) \leqslant t \text { and } g(y) \geqslant c\} \quad \forall c \in \mathbb{R} .
$$

Since $\widehat{d}(x, y)$ represents the time needed to travel from $y$ to $x$ with speed $v$, this new expression reads: $\bar{\Omega}_{t}^{c}$ is the union of all those points which can be visited by a particle starting from $y \in \bar{\Omega}_{0}^{c}$ with speed $v$ within time $t$.

REMARK 2.3 Assume that $\partial Q_{1}=\partial Q_{2}=J$. Then $v^{*}$ and $v_{*}$ are independent of $v_{J}$. Consequently, according to Definition 1 , no matter what value of $v_{J} \in(1,2)$ we take, the corresponding function $\varphi$ we constructed is always a viscosity solution to a fixed initial value problem, say corresponding to $v_{J}=3 / 2$. Thus, when the measure of $J$ is non-zero, non-uniqueness is expected. Even just for this particular example, it is not clear what should we substitute for $v$ when defining subsolutions and supersolutions in Definition 1, to distinguish these different viscosity solutions. (Clearly, the replacement of $v$ by $\limsup _{\delta \searrow 0}\left|B_{\delta}(x)\right|^{-1} \int_{B_{\delta}(x)} v$ for subsolutions and a similar replacement for supersolutions does not work.)

In $\S 5$, we shall prove uniqueness of the viscosity solution when $J$ consists of lower dimensional piecewise smooth hypersurfaces which are not tangent to each other at points of their intersection.

\section{Existence: Proof of Theorem 1}

We obtain a viscosity solution from a standard procedure: first regularize the problem, then establish a uniform estimate, and finally take the limit along a sequence and verify that the limit is a solution. The central difficulty here is the uniform estimate, for which we have to assume that $\ln v(x, t)-\ln m(t)$ is non-increasing in $t$ for some positive increasing function $m$. Another difficulty is the interpretation of the limit as a viscosity solution, for which we use Definition 1 for viscosity solutions.

For the reader's convenience, we divide the proof into three steps.

STEP 1. We regularize (1.1) by

$$
\begin{aligned}
\varphi_{t}^{\varepsilon}(x, t) & =v^{\varepsilon}(x, t)\left\{H^{\varepsilon}\left(\nabla \varphi^{\varepsilon}\right)+\varepsilon \Delta \varphi^{\varepsilon}\right\}, & & x \in \mathbb{R}^{N}, t>0, \\
\varphi^{\varepsilon}(x, 0) & =\varphi_{0}^{\varepsilon}(x), & & x \in \mathbb{R}^{N},
\end{aligned}
$$


where $v^{\varepsilon}, H^{\varepsilon}$ and $\varphi_{0}^{\varepsilon}$ are smooth regularizations of $v, H$, and $\varphi_{0}$ with the basic property that as $\varepsilon \searrow 0, v^{\varepsilon} \rightarrow v$ a.e. in $\mathbb{R}^{N} \times(0, \infty)$, and $H^{\varepsilon} \rightarrow H$ and $\varphi_{0}^{\varepsilon} \rightarrow \varphi_{0}$ uniformly in any compact subset of $\mathbb{R}^{N}$. Additional properties of $v^{\varepsilon}, H^{\varepsilon}$ and $\varphi_{0}^{\varepsilon}$ are listed as follows:

(a) for every $q \in \mathbb{R}^{N}$ and $\varepsilon>0,3 \sqrt{\varepsilon}+|q| / C \leqslant H^{\varepsilon}(q) \leqslant C(1+|q|)+3 \sqrt{\varepsilon}$ where $C$ is as in the theorem;

(b) for every $\varepsilon>0,\left\|\varphi_{0}^{\varepsilon}\right\|_{C^{2}\left(\mathbb{R}^{N}\right)} \leqslant 2 / N \sqrt{\varepsilon}$ and $\sup _{\mathbb{R}^{N}}\left|\nabla \varphi_{0}^{\varepsilon}\right| \leqslant \operatorname{ess} \sup _{\mathbb{R}^{N}}\left|\nabla \varphi_{0}\right|$;

(c) for every $\varepsilon>0$ and a.e. $x \in \mathbb{R}^{n}, \ln v^{\varepsilon}(x, t)-m^{\varepsilon}(t)$ is non-increasing in $t \in[0, \infty)$ where $m^{\varepsilon}(t)$ is a regularization of $m$ satisfying $\left\|m^{\varepsilon}\right\|_{C^{0}([0, T])} \leqslant\|m\|_{L^{\infty}(0, T)}$ for all $T>0$; also

$$
\underset{B_{\varepsilon}(x) \times(t-\varepsilon, t+\varepsilon)}{\operatorname{essinf}} v \leqslant v^{\varepsilon}(x, t) \leqslant \operatorname{ess}_{B_{\varepsilon}(x) \times[t-\varepsilon, t+\varepsilon]} v \quad \forall(x, t) \in \mathbb{R}^{N} \times[0, \infty) .
$$

In fact, $\varphi_{0}^{\varepsilon}$ can be obtained by mollifying $\min \left\{1 / N \sqrt{\varepsilon}, \max \left\{-1 / N \sqrt{\varepsilon}, \varphi_{0}\right\}\right\}, H^{\varepsilon}$ by mollifying $H+3 \sqrt{\varepsilon}$, whereas $v^{\varepsilon}$ can be obtained by taking the exponential of the mollification of $\ln v$, and $m^{\varepsilon}$ by the mollification of $m$, with the same mollifier as that for $\ln v$.

Set $C^{*}=\operatorname{ess} \sup v$. Then $\pm 2 / \sqrt{\varepsilon} \pm C^{*} H^{\varepsilon}(0) t$ is a super/sub solution to 3.1 , so by a standard theory of semilinear parabolic equations, $\sqrt{3.1}$ admits a unique global in time solution, which is bounded (with bounds depending on $\varepsilon$ ) in $C^{3}\left(\mathbb{R}^{N} \times[0, T]\right)$ for any $T>0$; see, for example, [18].

STEP 2. To pass to the limit, we need a few $\varepsilon$-independent estimates.

First we establish a uniform (in $\varepsilon$ ) bound for $\varphi_{t}^{\varepsilon}$. Note that (a) and (b) imply $H^{\varepsilon}\left(\nabla \varphi_{0}^{\varepsilon}\right)+\varepsilon \Delta \varphi_{0}^{\varepsilon}$ $>0$, so that $\varphi_{t}^{\varepsilon}(\cdot, 0)>0$ by evaluating (3.1) at $t=0$. Differentiating (3.1) with respect to $t$ yields

$$
\left(\varphi_{t}^{\varepsilon}\right)_{t}=v^{\varepsilon}\left[\nabla_{q} H^{\varepsilon} \cdot \nabla \varphi_{t}^{\varepsilon}+\varepsilon \Delta \varphi_{t}^{\varepsilon}\right]+\left(\ln v^{\varepsilon}\right)_{t} \varphi_{t}^{\varepsilon}
$$

where we have replaced the term $v_{t}^{\varepsilon}\left(H^{\varepsilon}+\varepsilon \Delta \varphi^{\varepsilon}\right)$ by $\left(\ln v^{\varepsilon}\right)_{t} \varphi_{t}^{\varepsilon}$ after using 3.1). Applying the maximum principle to the function $\varphi_{t}^{\varepsilon}$ then yields $\varphi_{t}^{\varepsilon}>0$ in $\mathbb{R}^{N} \times[0, \infty)$.

Set $w=\varphi_{t}^{\varepsilon} \exp \left(-m^{\varepsilon}(t)\right)$. Then (3.3) gives $w_{t}=v^{\varepsilon}\left[\nabla_{q} H^{\varepsilon} \nabla w+\varepsilon \Delta w\right]+\left(\ln v^{\varepsilon}-m^{\varepsilon}\right)_{t} w \leqslant$ $v^{\varepsilon}\left(\nabla_{q} H^{\varepsilon} \nabla w+\varepsilon \Delta w\right)$ since $\ln v^{\varepsilon}-m^{\varepsilon}$ is non-increasing in $t$. Therefore, the maximum principle implies that $w$ can only attain its maximum at $t=0$. Hence,

$$
0<\varphi_{t}^{\varepsilon}(x, t) \leqslant\left\|v^{\varepsilon}(\cdot, 0)\left[H^{\varepsilon}\left(\nabla \varphi_{0}^{\varepsilon}\right)+\varepsilon \Delta \varphi_{0}^{\varepsilon}\right]\right\|_{L^{\infty}\left(\mathbb{R}^{N}\right)} e^{m^{\varepsilon}(t)-m^{\varepsilon}(0)} \quad \forall x \in \mathbb{R}^{N}, t \geqslant 0 .
$$

Next we estimate the $L^{\infty}$ bound for $\left|\nabla \varphi^{\varepsilon}\right|$. For any fixed $t$, define $\Phi(y)=\frac{1}{\varepsilon} \varphi^{\varepsilon}(\varepsilon y, t)$. Then (3.1) gives

$$
\Delta_{y} \Phi+H^{\varepsilon}\left(\nabla_{y} \Phi\right)=f(y):=\varphi_{t}^{\varepsilon} / v^{\varepsilon} .
$$

Since $H^{\varepsilon}(q)$ grows linearly in $q$, applying local elliptic estimates [16] to [3.5], we get

$$
\left\|\nabla_{y} \Phi\right\|_{C^{0}\left(\mathbb{R}^{N}\right)}=\sup _{y \in \mathbb{R}^{N}}\left\|\nabla_{y} \Phi\right\|_{C^{0}\left(B_{1 / 2}(y)\right)} \leqslant C_{0}\left[\|f\|_{L^{\infty}\left(\mathbb{R}^{N}\right)}+\sup _{y \in \mathbb{R}^{N}}\left\|\nabla_{y} \Phi\right\|_{L^{1}\left(B_{1}(y)\right)}\right]
$$

where $C_{0}$ is a constant depending only on the space dimension $N$ and the linear growth rate of $H$.

We now estimate $\left\|\nabla_{y} \Phi\right\|_{L^{1}\left(B_{1}(y)\right)}$. Let $y \in \mathbb{R}^{N}$ be any fixed point. Set $F(r)=\int_{B_{r}(y)}\left|\nabla_{y} \Phi\right|$. Then, for any $r>1$,

$$
\begin{aligned}
\frac{\mathrm{d}}{\mathrm{d} r} F(r) & =\int_{\partial B_{r}(y)}\left|\nabla_{y} \Phi\right| \mathrm{d} S \geqslant-\int_{\partial B_{r}(y)} \partial_{n} \Phi \mathrm{d} S \\
& =-\int_{B_{r}(y)} \Delta_{y} \Phi=\int_{B_{r}(y)}\left\{H^{\varepsilon}\left(\nabla_{y} \Phi\right)-f\right\} \geqslant \frac{1}{C} F(r)-\omega_{N} r^{N}\|f\|_{L^{\infty}\left(\mathbb{R}^{N}\right)}
\end{aligned}
$$


where $\omega_{N}$ is the volume of the unit ball in $\mathbb{R}^{N}$. Multiplying by an integrating factor $e^{(-r+1) / C}$ and integrating the resulting inequality over $[1, r)$ we obtain

$$
e^{(-r+1) / C} F(r)-F(1) \geqslant-\omega_{N} \int_{1}^{r} s^{N} e^{(-s+1) / C} \mathrm{~d} s\|f\|_{\infty} \quad \forall r>1 .
$$

Letting $r \rightarrow \infty$ and using the fact that $F(r)=O\left(r^{N}\right)$, we deduce that

$$
\left\|\nabla_{y} \Phi\right\|_{L^{1}\left(B_{1}(y)\right)}=F(1) \leqslant C_{1}\|f\|_{L^{\infty}\left(\mathbb{R}^{N}\right)}, \quad C_{1}:=\omega_{N} \int_{0}^{\infty} s^{N} e^{(-s+1) / C} \mathrm{~d} s .
$$

Substituting this estimate into [3.6, we obtain

$$
\left\|\nabla \varphi^{\varepsilon}(\cdot, t)\right\|_{L^{\infty}\left(\mathbb{R}^{N}\right)}=\|\nabla \Phi\|_{C^{0}\left(\mathbb{R}^{N}\right)} \leqslant C_{0}\left[1+C_{1}\right]\|f\|_{L^{\infty}\left(\mathbb{R}^{N}\right)} \leqslant \frac{C_{0}\left[1+C_{1}\right]}{C_{*}}\left\|\varphi_{t}^{\varepsilon}(\cdot, t)\right\|_{L^{\infty}\left(\mathbb{R}^{N}\right)}
$$

by the definition of $f$. Here $C_{*}=\operatorname{ess} \inf v$.

Combining this with the estimate from 3.4], we obtain, for every $T>0$,

$$
\left\|\varphi_{t}^{\varepsilon}, \nabla \varphi^{\varepsilon}\right\|_{L^{\infty}\left(\mathbb{R}^{N} \times[0, T]\right)} \leqslant C_{2}\left\|H\left(\nabla \varphi_{0}\right)\right\|_{L^{\infty}\left(\mathbb{R}^{N}\right)} e^{\|m\|_{L^{\infty}[0, T]}}+o(1)
$$

where $C_{2}$ is independent of $\varepsilon$ and $o(1) \rightarrow 0$ as $\varepsilon \rightarrow 0$.

STEP 3. Now we take the limit of $\varphi^{\varepsilon}$ as $\varepsilon \searrow 0$ to obtain a viscosity solution.

Since $\varphi^{\varepsilon}(0,0)=\varphi_{0}^{\varepsilon}(0)$ is bounded uniformly in $\varepsilon$, 3.7. implies that the family $\left\{\varphi^{\varepsilon}\right\}_{0<\varepsilon<1}$ is a bounded set in $C^{1}\left(B_{R}(0) \times[0, T]\right)$ for any positive $R$ and $T$. Hence, there exists a function $\varphi$ and a subsequence of $\left\{\varphi^{\varepsilon}\right\}$, which we still denote by $\left\{\varphi^{\varepsilon}\right\}$, such that, as $\varepsilon \searrow 0$,

$$
\varphi^{\varepsilon} \rightarrow \varphi \quad \text { uniformly on any compact subset of } \mathbb{R}^{N} \times[0, \infty) .
$$

It is clear that $\varphi$ also satisfies the estimate 3.7$)$ with the $o(1)$ being removed.

We claim that $\varphi$ is a viscosity solution to $(1.1)$. First we verify that $\varphi$ is a subsolution in $\mathbb{R}^{N} \times$ $[0, T]$ for any fixed $T>0$. For any smooth function $\zeta(x, t)$ such that $\varphi-\zeta$ takes a strict local maximum in $\mathbb{R}^{N} \times[0, T]$ at $\left(x_{0}, t_{0}\right)$ with $t_{0} \in(0, T]$, the function $\varphi^{\varepsilon}(x, t)-\zeta(x, t)$ takes a local maximum in $\mathbb{R}^{N} \times[0, T]$ at $\left(x^{\varepsilon}, t^{\varepsilon}\right)$. By $\left.\sqrt{3.8}\right)$, we have $\lim _{\varepsilon \rightarrow 0}\left(x^{\varepsilon}, t^{\varepsilon}\right)=\left(x_{0}, t_{0}\right)$, and therefore $0<t^{\varepsilon} \leqslant T$ if $\varepsilon$ is sufficiently small. Thus, $\nabla \varphi^{\varepsilon}\left(x^{\varepsilon}, t^{\varepsilon}\right)=\nabla \zeta\left(x^{\varepsilon}, t^{\varepsilon}\right), \varphi_{t}^{\varepsilon}\left(x^{\varepsilon}, t^{\varepsilon}\right) \geqslant \zeta_{t}\left(x^{\varepsilon}, t^{\varepsilon}\right)$ and $\Delta \varphi^{\varepsilon}\left(x^{\varepsilon}, t^{\varepsilon}\right) \leqslant \Delta \zeta\left(x^{\varepsilon}, t^{\varepsilon}\right)$. Substituting these relations into 3.1 and letting $\varepsilon \rightarrow 0$, we conclude that $\varphi$ satisfies the condition for a subsolution. Similarly, we can show that $\varphi$ is a supersolution, so it is a viscosity solution. This completes the proof of Theorem 1 .

\section{Uniqueness under monotonicity property}

In this section, we prove the uniqueness of a viscosity solution when $v$ has the monotonicity property defined in Definition 2 in $§ 1$. For simplicity, we assume that $H$ is uniformly Lipschitz continuous with Lipschitz constant 1 . For a general Lipschitz function $H$, one can always replace it by $H(p) / C$ and replace $v$ by $C v$.

THEOREM 7 Assume that $H(\cdot)$ is non-negative and satisfies $|H(p)-H(q)| \leqslant|p-q|$ for all $p, q \in \mathbb{R}^{N}$. Also assume that $v$ is bounded and non-negative and has the monotonicity property at every point in $\mathbb{R}^{N} \times(0, \infty)$. Then Lipschitz continuous viscosity solutions to 1.1 are unique. 
Note that in this theorem $H(q)$ is not required to be monotonic in $|q|$. by

For convenience, in what follows we set $z=(x, t)$ and for $K>0$ we define a cone $C\left(x_{0}, t_{0}, K\right)$

$$
C\left(x_{0}, t_{0}, K\right)=\left\{(x, t): 0 \leqslant t \leqslant t_{0},\left|x-x_{0}\right| \leqslant K\left(t_{0}-t\right)\right\} .
$$

Theorem 7 follows from the following more general result.

Lemma 8 (Cone determination) Let $H$ be as in Theorem $7 z_{0}=\left(x_{0}, t_{0}\right)$ be any fixed point in $\mathbb{R}^{N} \times(0, \infty), v_{1}$ and $v_{2}$ be two bounded non-negative functions, and $K>1$ be any constant such that $K \geqslant \sup _{C\left(z_{0}, K\right)} v_{1}^{*}$. Assume that for every $\left(x^{*}, t^{*}\right) \in C\left(z_{0}, K\right)$, there exists a unit vector $\vec{e}=\vec{e}\left(x^{*}, t^{*}\right) \in \mathbb{R}^{N+1}$ such that

$$
\lim _{\rho \searrow 0+} \sup _{\left|y-x^{*}\right| \leqslant K\left(t^{*}-\tau\right) \leqslant M \rho}\left[\left(v_{1}\right)^{*}(y, \tau)-\left(v_{2}\right)_{*}((y, \tau)-\rho \vec{e})\right] \leqslant 0 \quad \forall M>1 .
$$

Suppose that on $C\left(z_{0}, K\right), \varphi_{1}$ is a subsolution of $\varphi_{t}=v_{1} H(\nabla \varphi), \varphi_{2}$ is a supersolution of $\varphi_{t}=$ $v_{2} H(\nabla \varphi)$, and either $\varphi_{1}$ or $\varphi_{2}$ is Lipschitz continuous. Then

$$
\max _{C\left(z_{0}, K\right)}\left\{\varphi_{1}-\varphi_{2}\right\}=\max _{C\left(z_{0}, K\right) \cap\{t=0\}}\left\{\varphi_{1}-\varphi_{2}\right\} .
$$

Proof. The proof is a careful modification of a classical comparison principle proof in [8].

By symmetry, we can assume without loss of generality that $\varphi_{2}$ is Lipschitz continuous and has been extended over $\mathbb{R}^{N} \times\left[0, t_{0}\right]$ with a Lipschitz constant denoted by $L$. Also, by taking smaller $t_{0}$ and a limit if necessary, we can assume that $\varphi_{2}$ is a viscosity supersolution on $C\left(x_{0}, t_{0}+o(1), K\right)$ where $o(1)>0$.

Suppose that the conclusion is not true. Then for some small $c>0$, the maximum of $\varphi_{1}(z)-$ $\varphi_{2}(z)-2 c t$ on $C\left(z_{0}, K\right)$ will be attained at some $z^{*}=\left(x^{*}, t^{*}\right) \in C\left(z_{0}, K\right)$ with $t^{*}>0$. We let $\vec{e}=\vec{e}\left(z^{*}\right)$ be as in the assumption and let

$$
h(x, t)=\left\{K^{2}\left(t^{*}-t\right)^{2}-\left|x-x^{*}\right|^{2}\right\}^{-1} .
$$

We define, for all sufficiently small positive $\varepsilon, \delta$, and $\rho$,

$$
G(z, \xi)=\varphi_{1}(z)-\varphi_{2}(\xi-\rho \vec{e})-c t-\delta h(z)-|z-\xi|^{2} / \varepsilon, \quad z=(x, t), \xi=(y, \tau) .
$$

Then the maximum of $G$ on $C\left(z^{*}, K\right) \times\left(\mathbb{R}^{N} \times\left[0, t^{*}\right]\right)$ is attained at some $\left(z_{\varepsilon}, \xi_{\varepsilon}\right)=$ $\left(\left(x_{\varepsilon}, t_{\varepsilon}\right),\left(y_{\varepsilon}, \tau_{\varepsilon}\right)\right)$ (which also depends on $\delta$ and $\rho$ ).

We first estimate the location of $\left(z_{\varepsilon}, \xi_{\varepsilon}\right)$. Since $h=\infty$ when $\left|x-x^{*}\right|=K\left|t-t^{*}\right|$, we have $\left|x_{\varepsilon}-x^{*}\right|<K\left(t^{*}-t_{\varepsilon}\right)$ and $0 \leqslant t_{\varepsilon}<t^{*}$. Also, evaluating $G\left(z_{\varepsilon}, \xi_{\varepsilon}\right) \geqslant G\left(z_{\varepsilon}, \xi\right)$ at $\xi=(1-s) z_{\varepsilon}+s \xi_{\varepsilon}$ with $s \in(0,1)$ gives

$$
\frac{1}{\varepsilon}\left(1-s^{2}\right)\left|z_{\varepsilon}-\xi_{\varepsilon}\right|^{2} \leqslant \varphi_{2}\left((1-s) z_{\varepsilon}+s \xi_{\varepsilon}-\rho \vec{e}\right)-\varphi_{2}\left(\xi_{\varepsilon}-\rho \vec{e}\right) .
$$

Using the Lipschitz continuity of $\varphi_{2}$ to estimate the right hand side and sending $s \nearrow 1$, we then obtain $2\left|z_{\varepsilon}-\xi_{\varepsilon}\right| \leqslant L \varepsilon$. Consequently, for any small $\delta>0, G\left(x^{*}, t^{*}-\delta^{1 / 3}, x^{*}, t^{*}-\delta^{1 / 3}\right) \leqslant$ 
$G\left(z_{\varepsilon}, \xi_{\varepsilon}\right) \leqslant G\left(z_{\varepsilon}, z_{\varepsilon}\right)+L^{2} \varepsilon / 2$. Therefore, since $\varphi_{1}(z)-\varphi_{2}(z)-2 c t$ attains its maximum at $z^{*}$,

$$
\begin{aligned}
c\left(t^{*}-t_{\varepsilon}\right)= & \left\{\left[\varphi_{1}\left(z_{\varepsilon}\right)-\varphi_{2}\left(z_{\varepsilon}\right)-2 c t_{\varepsilon}\right]-\left[\varphi_{1}\left(z^{*}\right)-\varphi_{2}\left(z^{*}\right)-2 c t^{*}\right]\right\} \\
& +\left\{G\left(x^{*}, t^{*}-\delta^{1 / 3}, x^{*}, t^{*}-\delta^{1 / 3}\right)-G\left(z_{\varepsilon}, z_{\varepsilon}\right)\right\} \\
& +\left\{\left[\varphi_{2}\left(z_{\varepsilon}\right)-\varphi_{2}\left(z_{\varepsilon}-\rho \vec{e}\right)\right]-\left[\varphi_{2}\left(z^{*}\right)-\varphi_{2}\left(z^{*}-\rho \vec{e}-\left(0, \delta^{1 / 3}\right)\right)\right]\right\} \\
& +\left\{\delta h\left(x^{*}, t^{*}-\delta^{1 / 3}\right)-\delta h\left(z_{\varepsilon}\right)-c \delta^{1 / 3}\right\}+\left\{\varphi_{1}\left(z^{*}\right)-\varphi_{1}\left(x^{*}, t^{*}-\delta^{1 / 3}\right)\right\} \\
\leqslant & 0+L^{2} \varepsilon / 2+\left\{L \rho+L\left(\rho+\delta^{1 / 3}\right)\right\}+\frac{1}{K^{2}} \delta^{1 / 3}+\left[\varphi_{1}\left(z^{*}\right)-\varphi_{1}\left(x^{*}, t^{*}-\delta^{1 / 3}\right)\right] .
\end{aligned}
$$

It then follows that $t_{\varepsilon} \rightarrow t^{*}$ as $\varepsilon, \delta, \rho \searrow 0$. In addition, by the estimates $\left|x_{\varepsilon}-x^{*}\right|<K\left|t_{\varepsilon}-t^{*}\right|$ and $\left|z_{\varepsilon}-\xi_{\varepsilon}\right| \leqslant L \varepsilon / 2$, we conclude that $z_{\varepsilon}$ is an interior point of $C\left(z_{0}, K\right), \xi_{\varepsilon}$ is an interior point of $C\left(x_{0}, t_{0}+o(1), K\right)$, and $\left(z_{\varepsilon}, \xi_{\varepsilon}\right) \rightarrow\left(z^{*}, z^{*}\right)$ as $\varepsilon, \delta, \rho \searrow 0$.

Note that with $\zeta(z)=\varphi_{2}\left(\xi_{\varepsilon}-\rho \vec{e}\right)+c t+\delta h(z)+\left|z-\xi_{\varepsilon}\right|^{2} / \varepsilon, \varphi_{1}(z)-\zeta(z)=G\left(z, \xi_{\varepsilon}\right)$ attains a local maximum at $z=z_{\varepsilon}$, and with $\widetilde{\zeta}(\xi)=\varphi_{1}\left(z_{\varepsilon}\right)-c t_{\varepsilon}-\delta h\left(z_{\varepsilon}\right)-\left|z_{\varepsilon}-\xi\right|^{2} / \varepsilon, \varphi_{2}(\xi-\rho \vec{e})-\widetilde{\zeta}(\xi)=$ $-G\left(z_{\varepsilon}, \xi\right)$ attains a local minimum at $\xi=\xi_{\varepsilon}$. The definition of subsolutions and supersolutions then gives

$$
\begin{aligned}
& c+\frac{2}{\varepsilon}\left(t_{\varepsilon}-\tau_{\varepsilon}\right)+K^{2} b \leqslant\left(v_{1}\right)^{*}\left(z_{\varepsilon}\right) H\left(\frac{2\left(x_{\varepsilon}-y_{\varepsilon}\right)}{\varepsilon}+\frac{b\left(x_{\varepsilon}-x^{*}\right)}{t^{*}-t_{\varepsilon}}\right), \\
&-\frac{2}{\varepsilon}\left(\tau_{\varepsilon}-t_{\varepsilon}\right) \geqslant\left(v_{2}\right)_{*}\left(\xi_{\varepsilon}-\rho \vec{e}\right) H\left(-\frac{2\left(y_{\varepsilon}-x_{\varepsilon}\right)}{\varepsilon}\right),
\end{aligned}
$$

where $b:=2 \delta h^{2}\left(x_{\varepsilon}, t_{\varepsilon}\right)\left(t^{*}-t_{\varepsilon}\right) \in(0, \infty)$. It then follows that

$$
\begin{aligned}
c \leqslant & {\left[\left(v_{1}\right)^{*}\left(z_{\varepsilon}\right)-\left(v_{2}\right)_{*}\left(\xi_{\varepsilon}-\rho \vec{e}\right)\right] H\left(\frac{2\left(x_{\varepsilon}-y_{\varepsilon}\right)}{\varepsilon}\right) } \\
& +\left(v_{1}\right)^{*}\left(z_{\varepsilon}\right)\left\{H\left(\frac{2\left(x_{\varepsilon}-y_{\varepsilon}\right)}{\varepsilon}+\frac{b\left(x_{\varepsilon}-x^{*}\right)}{t^{*}-t_{\varepsilon}}\right)-H\left(\frac{2\left(x_{\varepsilon}-y_{\varepsilon}\right)}{\varepsilon}\right)\right\}-K^{2} b \\
\leqslant & {\left[\left(v_{1}\right)^{*}\left(z_{\varepsilon}\right)-\left(v_{2}\right)_{*}\left(\xi_{\varepsilon}-\rho \vec{e}\right)\right] \sup _{|p| \leqslant L} H(p), }
\end{aligned}
$$

where the term on the second line is non-positive since $\left|x_{\varepsilon}-x^{*}\right| \leqslant K\left(t^{*}-t_{\varepsilon}\right), H$ is Lipschitz continuous with Lipschitz constant 1 , and $K \geqslant \operatorname{ess} \sup v_{1}^{*}$.

Finally, letting $\varepsilon \searrow 0$ along a subsequence such that $\left(z_{\varepsilon}, \xi_{\varepsilon}\right) \rightarrow\left(z_{\delta}^{\rho}, z_{\delta}^{\rho}\right)$, where $z_{\delta}^{\rho} \in$ $C\left(x^{*}, t^{*}, K\right)$, we obtain

$$
c \leqslant\left[\left(v_{1}\right)^{*}\left(z_{\delta}^{\rho}\right)-\left(v_{2}\right)_{*}\left(z_{\delta}^{\rho}-\rho \vec{e}\right)\right] \sup _{|p| \leqslant L} H(p) .
$$

First letting $\delta \searrow 0$ along a subsequence and then letting $\rho \searrow 0$ and using the estimate for $t-t_{\varepsilon}$ in (4.2) we obtain

$$
c \leqslant \sup _{|p| \leqslant L} H(p) \lim _{\rho \searrow 0} \sup _{\left|x-x^{*}\right| \leqslant K\left(t^{*}-t\right) \leqslant 2 K L \rho / c}\left[\left(v_{1}\right)^{*}(z)-\left(v_{2}\right)_{*}(z-\rho \vec{e})\right] .
$$

But this contradicts the assumption 4.1 . 


\section{Blow-up property}

To better understand the blow-up property in Definition 3, in this section we give a few more examples and list a few properties of the blow-up functions.

First we note that if $w(e):=\lim _{\lambda \searrow 0} v\left(z_{0}+\lambda e\right)$ exists for a.e. $e \in \mathbb{R}^{m}$, then it is clear that $w^{*}(e) \leqslant \lim \sup _{\lambda \searrow 0, y \rightarrow e} v^{*}\left(z_{0}+\lambda y\right)$ for every $e \in \mathbb{R}^{m}$. Nevertheless, the reverse inequality may not be true for every $e \in \mathbb{R}^{m}$, as demonstrated by Example 1.3. The requirement of the equalities in (1.4) for the blow-up asks the limit function not to lose by the a.e. sense the information concerning the ess sup and ess inf of the original function in cones $\left\{z_{0}\right\}+\left\{\rho \vec{e}: 0<\rho<r,\left|\vec{e}-\vec{e}_{0}\right| \leqslant \delta\right\}$ for $r \ll 1, \delta \ll 1$, and all $\vec{e}_{0} \in S^{N-1}$. Therefore, the existence of a first order blow-up not only requires $v(z)$ to have vanishing oscillation as $z$ approaches $z_{0}$ from almost every direction, but also prohibits essential cusps in the discontinuity set of $v$. Here essential cusp means that the esssup or essinf at the tip of the cusp is beyond the esssup or essinf of any of the nearby cones at the tip.

EXAMPLE 5.1 In $\mathbb{R}^{1}$, if $v$ has the blow-up property at every point, then $v\left(x_{0} \pm\right):=\lim _{x \rightarrow x_{0}^{ \pm}} v(x)$ exists for every $x_{0} \in \mathbb{R}^{1}$, so $v$ has only a jump discontinuity. The reverse is also true; namely, if $v$ has only a jump discontinuity, then $v$ has the blow-up property. They are even equivalent to the monotonicity property. Note that in the one-dimensional case, no geometry is involved, so condition (1.4) is automatic.

EXAMPLE 5.2 In $\mathbb{R}^{2}$, let $v=1$ when $y>x^{2}, v=3$ when $0<y \leqslant x^{2}, v=2$ when $-x^{2}<y \leqslant$ 0 , and $v=4$ when $y \leqslant-x^{2}$. Then $v$ has the blow-up property since the cusp of the discontinuity set $\left\{(x, y):|y|=x^{2}\right.$ or $\left.y=0\right\}$ at the origin is not essential.

Notice that if we switch 2 and 4, then the cusp of the discontinuity set becomes essential.

The existence of higher order blow-ups essentially requires that the function $v$, modulo a vanishing error, is constant in finitely many open cones with vertex at $z_{0}$, where the complement of the union of these open cones consists of lower dimensional hypersurfaces.

EXAMPLE 5.3 In $\mathbb{R}^{2}$, assume that $v$ has the blow-up property at the origin. Then the first order blow-up $v^{(1)}(x)$ at the origin is homogeneous of degree zero (i.e. $v^{(1)}(\rho x)=v^{(1)}(x)$ for all $\left.\rho>0\right)$, so it is determined by a function on $S^{1}$, written as $v^{(1)}\left(r e^{i \theta}\right)=f(\theta)$. As $v$ has a second order blowup, it is easy to show that $f(\cdot)$ has a first order blow-up. Thus, $f$ has only a jump discontinuity.

Now we recover $v$ near the origin, within an error $\varepsilon$. Let $J^{\varepsilon}=\left\{\theta_{1}, \ldots, \theta_{K^{\varepsilon}}\right\}$ be all the points where the jump of $f$ is bigger than $\varepsilon / 2$. Then, modulo an error of $\varepsilon / 2, f$ is continuous in $S^{1} \backslash J^{\varepsilon}$, so that, by virtue of 1.4 , there exists $r^{\varepsilon}>0$ such that

$$
\begin{aligned}
& \left|v\left(\rho e^{i \theta}\right)-f(\theta)\right| \leqslant \varepsilon \quad \forall \rho \in\left(0, r^{\varepsilon}\right), \forall \theta \notin \bigcup_{j=1}^{K^{\varepsilon}}\left(\theta_{j}-\varepsilon / M_{\varepsilon}, \theta_{j}+\varepsilon / M_{e}\right), \\
& \min \left\{f\left(\theta_{j}^{+}\right), f\left(\theta_{j}^{-}\right)\right\}-\varepsilon \leqslant v\left(\rho e^{i \theta}\right) \leqslant \max \left\{f\left(\theta_{j}^{+}\right), f\left(\theta_{j}^{-}\right)\right\}+\varepsilon \\
& \forall \rho \in\left(0, r_{\varepsilon}\right), \forall \theta \in\left[\theta_{j}-\varepsilon / M_{\varepsilon}, \theta_{j}+\varepsilon / M_{\varepsilon}\right], \forall j \quad\left(M_{\varepsilon}:=\min _{i \neq j}\left|\theta_{i}-\theta_{j}\right|\right) .
\end{aligned}
$$

One can check that the above two conditions (for arbitrary $\varepsilon$ ) are also sufficient for $v$ to have the blow-up property. (Higher space dimensional cases can be discussed in a similar manner.)

EXAMPLE 5.4 In $\mathbb{R}^{m}$ with $m \geqslant 2$, let $\Pi_{1}, \ldots, \Pi_{K}$ be $m-1$-dimensional closed hypersurfaces dividing $\mathbb{R}^{m}$ into open domains $\Omega_{1}, \ldots, \Omega_{M}$. Assume that $v$ is uniformly continuous in each $\Omega_{i}$, 
that all hypersurfaces are locally graphs and at their intersection, they have tangent planes nontangent to each other. Let $z_{0} \in \mathbb{R}^{m}$ be any point. Then we have the following:

(a) If $z_{0}$ is on none of the hypersurfaces, then $v$ is continuous at $z_{0}$.

(b) If $z_{0}$ lies only on one of the hypersurfaces, then the monotonicity condition holds for $v$ at $z_{0}$, since all these hypersurfaces are locally graphs.

(c) If $z_{0}$ is on the intersection of at least two hypersurfaces, then $v$ has the blow-up property since all the hypersurfaces intersecting at $z_{0}$ have tangent planes at $z_{0}$, and these tangent planes are not tangent to each other. (Note that the discussion is still valid if the words "tangent planes" are replaced by "tangent cones".)

We now establish certain properties for the blow-up functions.

LEMMA 9 Let $k \geqslant 1$ and assume that $v$ has a $k$-th order blow-up $v^{(k)}(\cdot)$ at $\left[z_{0}, \ldots, z_{k-1}\right]$. Then the following holds:

(1) $v^{(k)}(\cdot)$ is homogeneous of degree zero, that is, $v^{(k)}(\lambda z)=v^{(k)}(z)$ for all $\lambda>0$ and a.e. $z \in \mathbb{R}^{m}$. Consequently, $\left(v^{(k)}\right)^{*}(0)=\operatorname{ess} \sup _{\mathbb{R}^{m}} v^{(k)}$ and $\left(v^{(k)}\right)_{*}(0)=\operatorname{essinf}_{\mathbb{R}^{m}} v^{(k)}$.

(2) Denote by $T$ the orthogonal projection from $\mathbb{R}^{m}$ to $\left(\operatorname{span}\left\{z_{1}, \ldots, z_{k-1}\right\}\right)^{\perp}$. Then $v^{(k)}(z)=$ $v^{(k)}(T z)$ for every $z \in \mathbb{R}^{m}$. Consequently, $v^{(k)}$ is a function of $m-l$ independent variables, where $l$ is the dimension of $\operatorname{span}\left\{z_{1}, \ldots, z_{k-1}\right\}$.

(3) If $z_{k-1}$ linearly depends on $z_{1}, \ldots, z_{k-2}$, then $v^{(k)}(\cdot)=v^{(k-1)}(\cdot)$ a.e.

(4) If $\operatorname{span}\left\{z_{1}, \ldots, z_{k-1}\right\}=\mathbb{R}^{m}$, then $v^{(k)}\left[z_{0}, \ldots, z_{k-1}\right](\cdot)$ is a constant function.

Proof. The first assertion follows from the definition.

We prove the second assertion by the induction argument. The case $k=1$ is automatically true since $T$ is an identity. Assume that the statement is true for $k=j$.

Denote by $\widehat{T}$ the orthogonal projection from $\mathbb{R}^{m}$ to $\left(\operatorname{span}\left\{z_{1}, \ldots, z_{j-1}\right\}\right)^{\perp}$. Then, by induction, $v^{(j)}(z)=v^{(j)}(\widehat{T} z)$ for every $z \in \mathbb{R}^{m}$. Let $z_{j}$ be any fixed vector in $\mathbb{R}^{m}$. Consider two cases: (i) $\widehat{T} z_{j}=0$, and (ii) $\widehat{T} z_{j} \neq 0$.

CASE (i). Note that $v^{(j)}\left(z_{j}+\lambda y\right)=v^{(j)}\left(\widehat{T} z_{j}+\lambda \widehat{T} y\right)=v^{(j)}(\lambda \widehat{T} y)=v^{(j)}(\widehat{T} y)=v^{(j)}(y)$ since $v^{(j)}$ is homogeneous of degree 0 . Sending $\lambda \searrow 0$ then yields $v^{(j)}(\cdot)=v^{(j+1)}(\cdot)$, and hence

$$
v^{(j+1)}(T z)=v^{(j)}(T z)=v^{(j)}(\widehat{T} z)=v^{(j)}(z)=v^{(j+1)}(z) .
$$

CASE (ii). Set $z_{j}^{\perp}=\widehat{T} z_{j}$ and $T$ the projection from $\mathbb{R}^{m}$ to the space $\left(\operatorname{span}\left\{z_{1}, \ldots, z_{j}\right\}\right)^{\perp}=$ $\left(\operatorname{span}\left\{z_{1}, \ldots, z_{j-1}, z_{j}^{\perp}\right\}\right)^{\perp}$. For every $y \in \mathbb{R}^{m}$, define $\widehat{T} y=a z_{j}^{\perp}+T y$. Then

$$
v^{(j)}\left(z_{j}+\lambda y\right)=v^{(j)}\left(\widehat{T} z_{j}+\lambda \widehat{T} y\right)=v^{(j)}\left((1+a \lambda) z_{j}^{\perp}+\lambda T y\right)=v^{(j)}\left(z_{j}^{\perp}+\frac{\lambda}{1+a \lambda} T y\right) .
$$

Sending $\lambda \searrow 0$, we obtain $v^{(j+1)}\left[z_{0}, z_{1}, \ldots, z_{j}\right](y)=v^{(j+1)}\left[z_{0}, \ldots, z_{j-1}, z_{j}^{\perp}\right](T y)$. Since $T^{2}=T$, we have

$$
v^{(j+1)}\left[z_{0}, \ldots, z_{j-1}, z_{j}\right](T y)=v^{(j+1)}\left[z_{0}, \ldots, z_{j-1}, z_{j}^{\perp}\right]\left(T^{2} y\right)=v^{(j+1)}\left[z_{0}, z_{1}, \ldots, z_{j}\right](y) .
$$

This completes the induction and also the proof of the second assertion.

The third assertion follows from case (i) in the preceding proof whereas the last assertion is a direct consequence of the second assertion. This completes the proof. 
From the proof of the lemma, one sees that any higher order blow-up of $v$ (if it exists) can always be reduced to either a constant function, or $v^{(k)}\left[z_{0}, \ldots, z_{k-1}\right](\cdot)$ for some $k \leqslant m$ and some $z_{1}, \ldots, z_{k-1}$ forming an orthonormal base.

\section{Uniqueness}

Now we prove the following comparison principle which implies the uniqueness Theorem 2.

THEOREM 10 Let $H(q)$ be non-negative, uniformly Lipschitz continuous in $\mathbb{R}^{N}$, with Lipschitz constant 1, and non-decreasing in $|q|$. Let $v$ be non-negative and uniformly bounded by $K$ in $\mathbb{R}^{N} \times$ $(0, \infty)$. Let $\left(x_{0}, t_{0}\right) \in \mathbb{R}^{N} \times(0, \infty)$ be any fixed point. Assume that, for any given point $\left(x^{*}, t^{*}\right) \in$ $C\left(x_{0}, t_{0}, K\right)$, either (a) $v$ has the monotonicity property at $\left(x^{*}, t^{*}\right)$, or (b) $v$ has the blow-up property at $\left(x^{*}, t^{*}\right)$ and its first order blow-up $v^{(1)}\left[\left(x^{*}, t^{*}\right)\right](x, t)$ is independent of $t$ when $K\left|x-x_{0}\right| \leqslant t_{0}-t$. Suppose that $\varphi_{1}$ and $\varphi_{2}$ are Lipschitz continuous on $C\left(x_{0}, t_{0}, K\right)$ and, in viscosity sense,

$$
\left(\varphi_{1}\right)_{t} \leqslant v^{*} H\left(\nabla \varphi_{1}\right), \quad\left(\varphi_{2}\right)_{t} \geqslant v_{*} H\left(\nabla \varphi_{2}\right) \quad \text { in } C\left(x_{0}, t_{0}, K\right) .
$$

Then

$$
\max _{C\left(x_{0}, t_{0}, K\right)}\left\{\varphi_{1}-\varphi_{2}\right\}=\max _{C\left(x_{0}, t_{0}, K\right) \cap\{t=0\}}\left\{\varphi_{1}-\varphi_{2}\right\} .
$$

Proof. For the reader's convenience, we divide our proof into three steps.

STEP I. Assume that the assertion is not true. Then there exists a positive constant $c$ such that, for $\psi_{1}:=\varphi_{1}-2 c t$ and $\psi_{2}:=\varphi_{2}+2 c t$, the maximum of $\psi_{1}-\psi_{2}$ in $C\left(x_{0}, t_{0}, K\right)$ will be attained at some point $\left(x^{*}, t^{*}\right) \in C\left(x_{0}, t_{0}, K\right)$ with $t^{*}>0$.

If at $\left(x^{*}, t^{*}\right), v$ has the monotonicity property, then repeating the proof of Lemma 8 , we obtain a contradiction.

Hence, we need only consider the case where $v$ has the blow-up property at $\left(x^{*}, t^{*}\right)$. Since the first order blow-up of $v$ at $\left(x^{*}, t^{*}\right)$ is independent of $t$ when $K|x| \leqslant-t$, for convenience, we denote it by $w(x)=v^{(1)}\left[\left(x^{*}, t^{*}\right)\right](x, t), x \in \mathbb{R}^{N}, t \leqslant-K|x|$.

For every $\lambda>0$ we define

$$
\Psi_{j}^{\lambda}(x, t):=\frac{1}{\lambda}\left\{\psi_{j}\left(x^{*}+\lambda x, t^{*}+\lambda[t-1]\right)-\psi_{j}\left(x^{*}, t^{*}\right)\right\}, \quad j=1,2 .
$$

Then $\left\{\Psi_{j}^{\lambda}\right\}_{0<\lambda \leqslant 1 / t^{*}}(j=1,2)$ is a family of uniformly (in $\left.\lambda\right)$ Lipschitz continuous functions on $C(0,1, K)=\{(x, t): 0 \leqslant t \leqslant 1,|x| \leqslant K(1-t)\}$. Thus there exist Lipschitz continuous functions $\Psi_{1}$ and $\Psi_{2}$ on $C(0,1, K)$ such that, along a sequence $\lambda \searrow 0, \Psi_{j}^{\lambda} \rightarrow \Psi_{j}(j=1,2)$ uniformly on $C(0,1, K)$. Since, by the definition of viscosity sub-super solutions, $\left(\Psi_{1}^{\lambda}\right)_{t} \leqslant\left(v^{\lambda}\right)^{*} H\left(\nabla \Psi_{1}^{\lambda}\right)-2 c$ and $\left(\Psi_{2}^{\lambda}\right)_{t} \geqslant\left(v^{\lambda}\right)_{*} H\left(\nabla \Psi_{2}^{\lambda}\right)+2 c$ in $C(0,1, K)$ where $v^{\lambda}(x, t)=v\left(x^{*}+\lambda x, t^{*}+\lambda(t-1)\right)$, we can apply Lemma 3 and use the definition of $w(x)=v^{(1)}\left[x^{*}, t^{*}\right](x, t)$ to deduce that

$$
\left\{\begin{array}{l}
0=\Psi_{1}(0,1)-\Psi_{2}(0,1) \geqslant \Psi_{1}(x, t)-\Psi_{2}(x, t) \quad \text { on } C(0,1, K), \\
\left(\Psi_{1}\right)_{t} \leqslant w^{*}(x) H\left(\nabla \Psi_{1}\right)-2 c \quad \text { in } C(0,1, K), \\
\left(\Psi_{2}\right)_{t} \geqslant w_{*}(x) H\left(\nabla \Psi_{2}\right)+2 c \quad \text { in } C(0,1, K) .
\end{array}\right.
$$

STEP II. To finish the proof, we need only show that 6.1 cannot hold. We shall use a dimension reduction argument; namely, we show that (6.1) is impossible when $w$ is a function of $\left(x_{1}, \ldots, x_{k}\right)$ only, for $k=0,1, \ldots, N$ respectively. 
When $k=0, w$ is a constant. It then follows from Lemma 8 that 6.1 cannot hold.

Now let $k \in\{1, \ldots, N\}$ be any given integer and assume that 6.1 cannot hold if $w$ is a function of $\left(x_{1}, \ldots, x_{k-1}\right)$ only. We shall prove that 6.1$)$ cannot hold if $w$ is a function of $\left(x_{1}, \ldots, x_{k}\right)$. For convenience, we write $x=\left(x^{\prime}, x^{\prime \prime}\right) \in \mathbb{R}^{k} \times \mathbb{R}^{N-k}$.

Let $h \in C^{\infty}([-1, \infty))$ be a fixed positive function satisfying (1) $h(r)=1$ when $r \in[-1,1]$, (2) $h(r)=2 / r$ when $r \geqslant 2$, and (3) $(r h(r))^{\prime} \geqslant 0$ for all $r>-1$.

Let $M$ be a large positive constant to be determined later by $c$ only. For every small $\varepsilon>0$, we define a diffeomorphism $x=F_{\varepsilon}(y)$ by

$$
F_{\varepsilon}\left(y^{\prime}, y^{\prime \prime}\right)=\left(0^{\prime}, y^{\prime \prime}\right)+\left\{1+M h\left(\frac{\left|y^{\prime}\right|}{\varepsilon}\right)\right\}\left(y^{\prime}, 0^{\prime \prime}\right) .
$$

Then $w\left(F_{\varepsilon}^{-1}(x)\right)=w(x)$ since $w(x)$ is a zero degree homogeneous function of $x^{\prime}$.

Denote by $y=F_{\varepsilon}^{-1}(x)$ the inverse of $x=F_{\varepsilon}(y)$, and define

$$
\Psi_{2}^{\varepsilon}(x, t)=\Psi_{2}\left(F_{\varepsilon}^{-1}(x), t\right)-c[t-1], \quad(x, t) \in C(0,1, K) .
$$

Then, as $F_{\varepsilon}^{-1}\left(B_{r}(0)\right) \subset B_{r}(0)$ for every ball $B_{r}(0), \Psi_{2}^{\varepsilon}$ is well-defined on $C(0,1, K)$. In addition, $\lim _{\varepsilon \searrow 0} \Psi_{2}^{\varepsilon}=\Psi_{2}-c(t-1)$ uniformly on $C(0,1, K)$. Furthermore, the chain rule shows that

$$
\begin{aligned}
\left|\nabla_{x} \Psi_{2}^{\varepsilon}(x, t)\right| & \leqslant\left.\left|\nabla_{y} \Psi_{2}(y, t)\right|\right|_{y=F_{\varepsilon}^{-1}(x)} \text { a.e. on } C(0,1, K), \\
\left|\nabla_{x^{\prime}} \Psi^{\varepsilon}\left(x^{\prime}, x^{\prime \prime}, t\right)\right| & \leqslant \frac{1}{1+M} \sup _{C(0,1, K)}\left|\nabla \Psi_{2}\right| \quad \text { if }\left|x^{\prime}\right| \leqslant \varepsilon .
\end{aligned}
$$

As $H(q)$ is non-decreasing in $|q|$ and $w\left(F_{\varepsilon}^{-1}(x)\right)=w(x)$, one can use Lemma 6 to verify that, in viscosity sense,

$$
\left(\Psi_{2}^{\varepsilon}\right)_{t} \geqslant w_{*}(x) H\left(\nabla \Psi_{2}^{\varepsilon}\right)+c \quad \text { in } C(0,1, K) .
$$

Let $(\widehat{x}, \widehat{t})$ be the point in $C(0,1, K)$ where $\Psi_{1}(x, t)-\Psi_{2}^{\varepsilon}(x, t)$ attains its maximum on $C(0,1, K)$. Since $\Psi_{1}(0,1)-\Psi_{2}^{\varepsilon}(0,1)=0$ and uniformly as $\varepsilon \searrow 0, \Psi_{1}(x, 0)-\Psi_{2}^{\varepsilon}(x, 0) \rightarrow$ $\left[\Psi_{1}(x, 0)-\Psi_{2}(x, 0)\right]-c \leqslant-c$ for all $|x| \leqslant K$, we see that $\widehat{t}>0$ for all sufficiently small $\varepsilon$.

Writing $\widehat{x}=\left(\widehat{x}^{\prime}, \widehat{x}^{\prime \prime}\right) \in \mathbb{R}^{k} \times \mathbb{R}^{N-k}$, we consider two cases: (i) $\widehat{x}^{\prime} \neq 0^{\prime}$; (ii) $\widehat{x}^{\prime}=0^{\prime}$.

In the first case (i), one can repeat Step I to conclude that 6.1 holds for some different $\widetilde{\Psi}_{1}, \widetilde{\Psi}_{2}, \widetilde{c}=c / 2$ and $\widetilde{w}(x)=w^{(1)}[\widehat{x}](x)$. One can assume, after a rotation if necessary, that $\widehat{x}^{\prime}=\left(0, \ldots, 0, \widehat{x}_{k}^{\prime}\right)$. As $w$ is homogeneous of degree 0 and depends on $x^{\prime}$ only, $\widetilde{w}(x)=$ $w^{(1)}\left[\widehat{x}^{\prime}\right](x)$ depends only on $\left(x_{1}, \ldots, x_{k-1}\right)$ (Lemma 9). Hence, by our induction argument, we get a contradiction.

STEP III. It remains to consider case (ii): $\widehat{x}^{\prime}=0$. By a translation if necessary, we can assume that $\widehat{x}^{\prime \prime}=0$ so that $\widehat{x}=0$. (Recall that $w$ depends only on $x^{\prime}$.) Also, by adding a constant to $\Psi_{2}$ if necessary, we can assume that

$$
0=\Psi_{1}(0, \widehat{t})-\Psi_{2}^{\varepsilon}(0, \widehat{t}) \geqslant \Psi_{1}(x, t)-\Psi_{2}^{\varepsilon}(x, t) \quad \text { in } C(0, \widehat{t}, K) .
$$

Let us consider $\Psi_{1}$ and $\Psi_{2}^{\varepsilon}$ in the set $C_{\varepsilon}=\left\{(x, t): \widehat{t}-\varepsilon^{2} \leqslant t \leqslant \widehat{t},|x| \leqslant K(\widehat{t}-t)\right\}$. Let $Z(x, t)$ be the viscosity solution to

$$
\begin{cases}Z_{t}=V H(\nabla Z)-c & \text { in } \left.\mathbb{R}^{N} \times \widehat{t}-\varepsilon^{2}, \widehat{t}\right], \\ Z=\Psi_{2}^{\varepsilon} & \text { on } \mathbb{R}^{N} \times\left\{\widehat{t}-\varepsilon^{2}\right\},\end{cases}
$$

where $V=\sup _{x \in \mathbb{R}^{N}} w^{*}(x)=w^{*}(0)$. Since $V$ is a constant, $Z$ exists and is unique. 
As $\Psi_{1}\left(\cdot, \widehat{t}-\varepsilon^{2}\right) \leqslant \Psi_{2}^{\varepsilon}\left(\cdot, \widehat{t}-\varepsilon^{2}\right)=Z\left(\cdot, \widehat{t}-\varepsilon^{2}\right)$ in $\left\{|x| \leqslant K \varepsilon^{2}\right\}$, applying the comparison Lemma 8 (with $v=V$ ) to $\Psi_{1}$ and $Z-c\left(t-\widehat{t}+\varepsilon^{2}\right)$ in $C_{\varepsilon}$ then gives $\Psi_{1}(0, \widehat{t}) \leqslant Z(0, \widehat{t})-c \varepsilon^{2}$.

Next, we compare $Z$ with $\Psi_{2}^{\varepsilon}$. For this purpose, we first estimate $\nabla_{x^{\prime}} Z$. For any $e \in \mathbb{R}^{k} \times\{0\}$, $Z(x+e, t)$ is also a viscosity solution of $Z_{t}=V H(\nabla Z)-c$, so that, by the comparison Lemma 8 , the maximum of $|Z(\cdot+e, t)-Z(\cdot, t)|$ in $C$ is attained at $t=\widehat{t}-\varepsilon^{2}$. Sending $\varepsilon \rightarrow 0$ and using the initial value of $Z$ at $t=\widehat{t}-\varepsilon^{2}$, we obtain, for every $(x, t) \in C,\left|\nabla_{x^{\prime}} Z(x, t)\right| \leqslant \sup _{C}\left|\nabla_{x^{\prime}} \Psi_{2}^{\varepsilon}\right| \leqslant$ $L /(1+M)$ by $(6.2)$ and the definition of $C$. It then follows from Lemma 5 that, in viscosity sense,

$$
Z_{t}=V H\left(\nabla_{x^{\prime}} Z, \nabla_{x^{\prime \prime}} Z\right)-c \leqslant V H\left(0, \nabla_{x^{\prime \prime}} Z\right)+\frac{V L}{1+M}-c \leqslant V H\left(0, \nabla_{x^{\prime \prime}} Z\right)
$$

if we take $M$ large enough (depending only on $c$ ). Here we assume that the Lipschitz constant of $H$ is 1 .

Now let $\left\{z^{m}\right\}_{m=1}^{\infty}$ be a sequence of points in $\mathbb{R}^{k}$ such that $\lim _{m \rightarrow \infty} z^{m}=0^{\prime}$ and

$$
\lim _{m \rightarrow \infty} w_{*}\left(z^{m}, 0^{\prime \prime}\right)=w^{*}(0)=V .
$$

Then, as a function of $\left(x^{\prime \prime}, t\right), \Psi_{2}^{\varepsilon}\left(z^{m}, x^{\prime \prime}, t\right)$ satisfies, in viscosity sense,

$$
\left(\Psi_{2}^{\varepsilon}\right)_{t} \geqslant w_{*}\left(z^{m}, 0^{\prime \prime}\right) H\left(0^{\prime}, \nabla_{x^{\prime \prime}} \Psi_{2}^{\varepsilon}\right)+c \geqslant V H\left(0^{\prime}, \nabla_{x^{\prime \prime}} \Psi_{2}^{\varepsilon}\right)
$$

for all $m$ large enough (depending only on $c$ ). Hence, by the comparison Lemma 8 .

$$
\Psi_{2}^{\varepsilon}\left(z^{m}, 0^{\prime \prime}, t\right) \geqslant Z\left(z^{m}, 0^{\prime \prime}, t\right), \quad t \in\left[\widehat{t}-\varepsilon^{2}, \widehat{t}-K\left|z^{m}\right|\right] .
$$

Sending $m$ to $\infty$ we then obtain $\Psi_{2}^{\varepsilon}(0, \widehat{t}) \geqslant Z(0, \widehat{t})$, which contradicts the assumption $0=$ $\Psi_{1}(0, \widehat{t})-\Psi_{2}^{\varepsilon}(0, \widehat{t})$ and our earlier comparison of $Z$ to $\Psi_{1}\left(\Psi_{1}(0, \widehat{t}) \leqslant Z(0, \widehat{t})-c \varepsilon^{2}\right)$.

In conclusion, 6.1) cannot hold. This completes the proof of the lemma.

REMARK 6.1 1) From the proof, one sees that only the restriction of $v$ to the cone $C\left(x^{*}, t^{*}, K\right)$ is needed. Hence, the alternative condition (b) in the requirement of the blow-up property at $\left(x^{*}, t^{*}\right)$ can be weakened to: the function $v$, after replacement by a constant outside of $C\left(x^{*}, t^{*}, K+1\right)$, has a first order blow-up $v^{(1)}\left[x^{*}, t^{*}\right](x, t)$, which is independent of $t$ when $K|x| \leqslant-t$, and if we denote by $w(x)$ the $t$ independent blow-up, then $w$ has the blow-up property at every $x \in \mathbb{R}^{N}$. One can further weaken this requirement on $w$ to: for any linearly independent unit vectors $e^{1}, \ldots, e^{N}$ in $\mathbb{R}^{N}$, there exists $k \in\{0,1, \ldots, N-1\}$ such that $w^{(k)}\left[e^{1}, \ldots, e^{k}\right](\cdot)$ exists and has the monotonicity property at $e^{k+1}$. The proof follows the same lines, by just adding, after the definition of $(\widehat{x}, \widehat{t})$ near the end of Step II: "If $w$ has the monotonicity property at $\widehat{x}$, then the proof of Lemma 8 applies."

2) If $v$ does not satisfy the assumption of Theorem 2 just on $\mathbb{R}^{N} \times\left\{t_{1}, \ldots, t_{k}\right\}$, then one can establish comparison successively in each interval $\left(0, t_{1}\right),\left(t_{1}, t_{2}\right), \ldots,\left(t_{k-1}, t_{k}\right)$, and $\left(t_{k}, \infty\right)$, and hence the assertion of the theorem is still valid in $\mathbb{R}^{N} \times(0, \infty)$.

3) In general if for any $\varepsilon>0$, there exists an open set $O$ which is a union of finitely many open intervals,

$$
O=\left(t_{1}, s_{1}\right) \cup\left(t_{2}, s_{2}\right) \cup \cdots \cup\left(t_{k}, s_{k}\right),
$$

such that $|O|<\varepsilon$ and $v$ satisfies the assumption of Theorem 2 on $[0, T] \backslash O$, then the assertion of the theorem is still valid on $\mathbb{R}^{N} \times[0, T]$. 
Indeed, the value of the solution $\varphi(x, t)$ for $t \in\left(s_{j}, t_{j+1}\right)$ is uniquely determined by the value at $t=s_{j}$, and continuously depends on the value at $t=s_{j}$ in a Lipschitz manner. By Lipschitz continuity, we have

$$
\sum_{j} \sup _{x \in \mathbb{R}^{N}}\left|\varphi\left(x, s_{j}\right)-\varphi\left(x, t_{j}\right)\right| \leqslant L \varepsilon .
$$

Thus the uniqueness of Lipschitz viscosity solutions follows.

\section{Acknowledgments}

X. Chen thanks for the financial support of the National Science Foundation Grant DMS-0203991. The authors would like to thank the referee for helpful suggestions. They would also like to thank Professor C. M. Elliott for the motivation of this research and many helpful discussions.

\section{REFERENCES}

1. Barron, E. N., \& LiU, W. Semicontinuous solutions for Hamilton-Jacobi equations and the $L^{\infty}$ control problem. Appl. Math. Optim. 34 (1996), 325-360. Zbl 0881.49019 MR 1408743

2. Brauner, C. M., Fife, P., Namah, G., \& Schmidt-Lainé, C. Propagation of a flame front in a striated solid medium: A homogenization analysis. Quart. Appl. Math. 51 (1993), 467-493. Zbl 0803.35009 MR 1233525

3. BRIAni, A., \& DAVIni, A. Monge solutions for discontinuous Hamiltonians. ESAIM Control Optim. Calc. Var. 11 (2005), 229-251. Zbl 1087.35023 MR 2141888

4. Camilli, F. An Hopf-Lax formula for a class of measurable Hamilton-Jacobi equations. Nonlinear Anal. 57 (2004), 265-286. Zbl 1051.35029 MR 2056431

5. CAmilli, F., \& Siconolfi, A. Hamilton-Jacobi equations with measurable dependence on the state variables. Adv. Differential Equations 8 (2003), 733-768. Zbl 1036.35052 MR 1969652

6. Chen, X. F., \& Namah, G. Wave propagation under curvature effect in a heterogeneous medium. Appl. Anal. 64 (1997), 219-233. Zbl 0878.35009 MR 1460080

7. Chen, Y. G., GigA, Y., \& Goto, S. Uniqueness and existence of viscosity solution of generalized mean curvature flow equations. J. Differential Geom. 33 (1991), 749-786. Zbl 0696.35087 MR 1100211

8. Crandall, M. G., Evans, L. C., \& LiOns, P.-L. Some properties of viscosity solutions of HamiltonJacobi equations. Trans. Amer. Math. Soc. 282 (1984), 487-502. Zbl 0543.35011 MR 0732102

9. CRAndall, M. G., Ishit, H., \& LiOns, P.-L. User's guide to viscosity solutions of second order partial differential equations. Bull. Amer. Math. Soc. 27 (1992), 1-67. Zbl 0755.35015 MR 1118699

10. Crandall, M. G., \& Lions, P.-L. Viscosity solutions of Hamilton-Jacobi equations. Trans. Amer. Math. Soc. 277 (1983), 1-42. Zbl 0599.35024 MR 0690039

11. Dafermos, C. M. Trend to steady state in a conservation law with spatial inhomogeneity. Quart. Appl. Math. 45 (1987), 313-319. Zbl 0665.76079 MR 0895101

12. Deckelnick, K., \& Elliott, C. M. Uniqueness and error analysis for Hamilton-Jacobi equations with discontinuities. Interfaces Free Bound. 6 (2004), 329-349. Zbl 1081.35115 MR 2095336

13. Deckelnick, K., \& Elliott, C. M. Propagation of graphs in two-dimensional inhomogeneous media. Appl. Numer. Math. 56 (2006), 1163-1178. Zbl 1126.65073 MR 2244969

14. Evans, L. C., \& SPRuck, J. Motion by mean curvature I. J. Differential Geom. 33 (1991), 635-681. Zbl 0726.53029

15. Giga, Y., Goto, S., \& Ishit, H. Global existence of weak solutions for interface equations coupled with diffusion equations. SIAM J. Math. Anal. 23 (1992), 821-835. Zbl 0754.35061 MR 1166559 
16. Gilbarg, D., \& Trudinger, N. S. Elliptic Partial Differential Equations of Second Order. Springer, New York, 1983. Zbl 0562.35001 MR 0737190

17. IsHII, H. Hamilton-Jacobi equations with discontinuous Hamiltonians on arbitrary open sets. Bull. Fac. Sci. Engrg. Chuo Univ. 28 (1985), 33-77. Zbl 0937.35505 MR 0845397

18. LAdYŽEnsKajA, O. A., SOlonNikov, V. A., \& URAL'CEVA, N. N. Linear and Quasilinear Equations of Parabolic Type. Amer. Math. Soc., Providence, RI (1968). Zbl 0174.15403 MR 0241822

19. Newcomb II, R. T., \& Su, J. Eikonal equations with discontinuities. Differential Integral Equations 8 (1995), 1947-1960. Zbl 0854.35022 MR 1348959

20. Osher, S., \& SethiAn, J. A. Fronts propagating with curvature-dependent speed: Algorithms based on Hamilton-Jacobi formulations. J. Comput. Phys. 79 (1988). 12-48. Zbl 0659.65132 MR 0965860

21. Soravia, P. Boundary value problems for Hamilton-Jacobi equations with discontinuous Lagrangian. Indiana Univ. Math. J. 51 (2002), 451-477. Zbl 1032.35055 MR 1909297

22. TOURIN, A. A comparison theorem for a piecewise Lipschitz continuous Hamiltonian and applications to shape-from-shading. Numer. Math. 62 (1992), 75-85. Zbl $0762.65087 \mid$ MR 1159046 